\title{
Dark matter monopoles, vectors and photons
}

\author{
Valentin V. Khoze and Gunnar Ro \\ IPPP, Department of Physics, Durham University, \\ South Road, Durham, U.K. \\ E-mail: valya.khoze@durham.ac.uk, g.o.i.ro@durham.ac.uk
}

AbStRaCT: In a secluded dark sector which is coupled to the Standard Model via a Higgs portal interaction we arrange for the existence of 't Hooft-Polyakov magnetic monopoles and study their implications for cosmology. We point out that a dark sector which can accommodate stable monopoles will also contain massless dark photons $\gamma^{\prime}$ as well as charged massive vector bosons $W_{ \pm}^{\prime}$. The dark matter in this scenario will be a combination of magnetically and electrically charged species under the unbroken U(1) subgroup of the dark sector. We estimate the cosmological production rate of monopoles and the rate of monopole-anti-monopole annihilation and conclude that monopoles with masses of few hundred $\mathrm{TeV}$ or greater, can produce sizeable contributions to the observed dark matter relic density. We scan over the parameter space and compute the relic density for monopoles and vector bosons. Turning to dark photons, we compute their contribution to the measured density of relativistic particles $N_{\text {eff }}$ and also apply observational constraints from the Bullet cluster and other large scale galaxies on long-range interactions of monopoles and of dark vector bosons. At scales relevant for dwarf galaxies we identify regions on the parameter space where self-interacting monopole and vector dark mater components can aid solving the core-vs-cusp and the too-big-to-fail problems.

Keywords: Beyond Standard Model, Cosmology of Theories beyond the SM, Solitons Monopoles and Instantons

ARXIV EPRINT: 1406.2291 


\section{Contents}

1 Introduction 1

1.1 The model 2

1.2 Monopoles 3

1.3 Mass-scale generation 3

2 Dark radiation and $\mathrm{N}_{\text {eff }} \quad 5$

3 Dark matter relic density $\quad 7$

3.1 Dark gauge bosons: Sommerfeld enhancement and relic density 7

$\begin{array}{lll}3.2 & \text { Dark monopoles } & 9\end{array}$

3.2.1 Production of monopoles 9

$\begin{array}{lll}3.2 .2 & \text { Evolution of monopoles } & 12\end{array}$

$\begin{array}{ll}3.2 .3 \text { Current density of monopoles } & 15\end{array}$

4 Self-interacting dark matter $\quad 18$

$\begin{array}{llr}5 & \text { Conclusions } & 20\end{array}$

A Coleman-Weinberg with an adjoint scalar 22

\section{Introduction}

A general and simple way to introduce dark matter into particle physics is to extend the Standard Model (SM) by a Dark sector, which includes dark matter particles, as well as possibly many other degrees of freedom.

If the Dark sector contains magnetic monopoles, can they be dark matter? The Dark sector, as the SM itself, can contain non-Abelian gauge fields and scalar fields, and if the Dark sector model is of the Georgi-Glashow type, 't Hooft-Polyakov monopoles [1, 2] will necessarily exist - this by itself of course does not mean that they have been or can be produced. These dark magnetic monopoles carry dark magnetic charge and are stable; they can also be relatively light, for example much lighter than the monopoles of Grand Unified theories.

The motivation of this paper is to investigate the cosmological consequences of magnetic monopoles of the dark sector. Can the monopoles contribute to the observed relic density and how sizeable can their contribution be; what was the cosmological production rate of monopoles and their annihilation rate; more importantly, which additional features emerge from the dark sector for it to be able to support monopoles? What are the cosmological and phenomenological constraints on the complete model and is it more attractive 
than the more traditional models of collisionless WIMP dark matter? These are some of the questions we want to address.

Models of dark matter with non-Abelian Dark sectors interacting with the visible SM sector only weakly, for example via some portal interactions is a popular approach to dark matter, see e.g. [3-5]. Cosmological production rate of light and heavy magnetic monopoles and their contributions to dark matter were discussed previously in [6]. We will incorporate these results in our analysis. More recently dark sector monopoles and vector bosons were considered in [7] with the authors of [7] concluding that the monopole contribution to the dark matter density should be negligible. This does not agree with our findings in section 3 .

Multi-component vector and scalar dark matter resulting from a non-Abelian Dark sector coupled to the SM via the Higgs portal interaction, was studied recently in [8] also with the view of helping to stabilise the SM Higgs potential, following earlier work $[9,10]$. We will now introduce magnetic monopoles into the theory.

\section{$1.1 \quad$ The model}

Consider the Standard Model extended by a hidden (a.k.a. Dark) sector which contains an $\mathrm{SU}(2)_{D}$ gauge group and a scalar field $\Phi$ in the adjoint representation of $\mathrm{SU}(2)_{D}$ (this is the simplest model of interest containing topologically stable monopoles; the Reader can imagine more complicated versions of the Dark sector(s), but the present model is fully adequate for our settings). The Lagrangian for the Dark sector is:

$$
\mathcal{L}_{D}=-\frac{1}{2} \operatorname{Tr} F_{\mu \nu}^{\prime} F^{\prime} \mu \nu+\operatorname{Tr}\left(D_{\mu} \Phi\left(D^{\mu} \Phi\right)^{\dagger}\right)-\lambda_{\phi} \operatorname{Tr}\left(\Phi \Phi^{\dagger}\right)^{2}+m^{2} \operatorname{Tr}\left(\Phi \Phi^{\dagger}\right), \quad \Phi=\phi_{a} \frac{\sigma_{a}}{2} .
$$

Here $F_{\mu \nu}^{\prime}$ is the field strength of the $\mathrm{SU}(2)_{D}$ gauge field $A_{\mu}^{\prime}=A_{\mu}^{\prime a} \frac{\sigma_{a}}{2}$, the covariant derivative is $D_{\mu} \Phi=\partial_{\mu} \Phi+i g_{D}\left[A_{\mu}^{\prime}, \Phi\right]$, where $g_{D}$ is the gauge coupling, and $\sigma_{a=1,2,3}$ are the Pauli matrices.

The $\Phi$-field also couples to the SM via the Higgs Portal interaction,

$$
\mathcal{L}_{\mathrm{HP}}=\lambda_{\mathrm{P}}\left(H^{\dagger} H\right) \operatorname{Tr}\left(\Phi \Phi^{\dagger}\right) .
$$

In the absence of other matter fields in the Dark sector, these are the only interactions between the SM and the Dark sector. In particular, there is no kinetic mixing between the non-Abelian Dark sector $\mathrm{SU}(2)_{D}$ and the SM gauge groups.

The scalar potential in our Dark-sector Lagrangian (1.1) contains the negative masssquared term, $-m^{2} \operatorname{Tr}\left(\Phi \Phi^{\dagger}\right)$, for the adjoint scalar. As the result, $\mathcal{L}_{D}$ has a non-trivial vacuum $\langle\Phi\rangle \neq 0$ which brakes the $\mathrm{SU}(2)_{D}$ gauge symmetry to $\mathrm{U}(1)_{D}$. Using gauge freedom we can set

$$
\langle\Phi\rangle=\left\langle\phi_{3}\right\rangle \frac{\sigma_{3}}{2}, \quad \text { where } \quad\left\langle\phi_{3}\right\rangle=w=m / \sqrt{\lambda_{\phi}} .
$$

After symmetry breaking in the Dark sector we get two massive gauge bosons $W_{ \pm}^{\prime}$ with mass $M_{W^{\prime}}=g_{D} w$, one massive scalar $m_{\phi}=\sqrt{2} m$ and one massless gauge boson $\gamma^{\prime}$. $\operatorname{SU}(2)_{D}$ has been broken to a massless $\mathrm{U}(1)_{D}$.

The effect of symmetry breaking is communicated from the Dark to the SM sector via the Higgs Portal interaction (1.2) which can generate the $\mu_{\mathrm{SM}}^{2}$ term in the SM effective 
potential,

$$
V(H)_{\mathrm{SM}}=-\frac{1}{2} \mu_{\mathrm{SM}}^{2} H H^{\dagger}+\lambda_{\mathrm{SM}}\left(H H^{\dagger}\right)^{2} .
$$

If $\mu_{\mathrm{SM}}^{2}$ was absent at tree level, the Dark sector generates the contribution $\mu_{\mathrm{SM}}^{2}=\lambda_{\mathrm{P}}\langle|\Phi|\rangle^{2}$ and triggers the electroweak symmetry breaking with the Higgs vev and mass,

$$
v=\frac{\mu_{\mathrm{SM}}}{\left(2 \lambda_{\mathrm{SM}}\right)^{1 / 2}} \simeq 246 \mathrm{GeV}, \quad m_{h \mathrm{SM}}=\mu_{\mathrm{SM}} \simeq 126 \mathrm{GeV} .
$$

\subsection{Monopoles}

It is well known that the spectrum of the $\mathrm{SU}(2)$ gauge theory with an adjoint scalar must contain 't Hooft-Polyakov magnetic monopoles [1, 2].

The mass of the monopoles is bounded from bellow by the Bogomolny bound [11],

$$
M_{\mathrm{m}} \geq \frac{4 \pi}{g_{D}} w=\frac{M_{W^{\prime}}}{\alpha_{D}},
$$

and monopoles have a magnetic charge $g_{\mathrm{mD}}=\frac{4 \pi}{g_{D}}$. For BPS monopoles in the limit where $\lambda_{\phi} \rightarrow 0$ the mass saturates the Bogomolny bound. More generally, away from the BPS limit, the monopole mass is given by $M_{\mathrm{m}}=\frac{M_{W^{\prime}}}{\alpha_{D}} f\left(\lambda_{\phi} / g_{D}^{2}\right)$ where $f$ is a smooth monotonically increasing function from $f(0)=1$ to $f(\infty) \simeq 1.787$, see e.g. [12]. Consequently we will use the Bogomolny bound as a reasonable approximation for the monopole mass for all values of $\lambda_{\phi}$.

\subsection{Mass-scale generation}

What is the origin of the $m^{2}$ term in (1.1)?

(1.) We can choose to make the full theory classically scale-invariant (CSI), in this case all input mass scales of the classical Lagrangian are set to zero, thus $m_{\mathrm{cl}}^{2} \equiv 0{ }^{1}$ The vacuum expectation value $\langle\Phi\rangle=w \neq 0$ is then generated radiatively via the Coleman-Weinberg (CW) mechanism [13]. In the appendix we outline how this works in massless Georgi-Glashow theory. The Dark gauge symmetry is broken by $\langle\Phi\rangle$ and this can be recast as generating an effective $m^{2}$ term in (1.1) in the CSI Standard Model $\times \mathrm{SU}(2)_{D}$ theory. This is a minimal scenario where dynamical mass generation occurs directly in the Dark sector, i.e. we have identified the mass-generating sector with the Dark sector, $\mathrm{SU}(2)_{\mathrm{CW}}=\mathrm{SU}(2)_{D}$.

(2.) A complimentary approach is to keep the mass-scale-generating sector and the Dark sector distinct. Then interactions between the two sectors would transmit the mass scale from the mass-scale-generating sector to the he Dark sector. For example, in CSI settings we can think of the $\mathrm{SM} \times \mathrm{SU}(2)_{D} \times G_{\mathrm{CW}}$ model, where $G_{\mathrm{CW}}$ is the ColemanWeinberg gauge sector which generates the vev $\left\langle\varphi_{\mathrm{CW}}\right\rangle$ for the CW scalar field. This radiatively generated scale is then transmitted to the Dark sector scalar and to

\footnotetext{
${ }^{1}$ We should make it clear that one can treat $m^{2}$ as an input parameter and not consider CSI at all, without invalidating any of the cosmological arguments that will follow.
} 
the SM Higgs field via scalar portal interactions, $\mathcal{L}_{\text {Portal }} \ni \lambda_{\mathrm{CWD}}\left|\varphi_{\mathrm{CW}}\right|^{2} \operatorname{Tr}\left(\Phi \Phi^{\dagger}\right)+$ $\lambda_{\mathrm{CWH}}\left|\varphi_{\mathrm{CW}}\right|^{2}\left(H^{\dagger} H\right)$ such that $\lambda_{\mathrm{CWD}}\left|\left\langle\varphi_{\mathrm{CW}}\right\rangle\right|^{2}=m^{2}$ in (1.1). ${ }^{2}$ In the above, $G_{\mathrm{CW}}$ is an example of the mass-generating sector, in general it does not have to be reliant on the CW mechanism, the mass scale can arise from any dimensional transmutation-type dynamical argument, including a strongly coupled sector.

Our reason for distinguishing between these two classes of models is the effect on the cosmological production of magnetic monopoles. The monopole production rate $[14,15]$ will depend on the nature of the phase transition in the Dark sector when the temperature in the early Universe falls below the critical temperature of $\mathrm{SU}(2)_{D}$. In the ColemanWeinberg sector the phase transition is of the first order, while in the Standard Model sector the electroweak phase transition is very weakly first order or second order [16-18]. The distinction can be traced to the value of the scalar self-coupling constant: in CW models $\lambda$ is small relative to the gauge coupling (resulting in CW scalar masses being 1-loop suppressed relative to $W^{\prime}$ masses), in the SM this is not the case, with the Higgs being heavier than $W$ and $Z$.

The Dark sector model (1.1) has three dark ingredients: dark photons $\gamma^{\prime}$, dark massive vector bosons $W_{ \pm}^{\prime}$, and dark magnetic monopoles $M_{\mathrm{mg} \pm}^{\prime}$. Massless $\gamma^{\prime}$ photons is the Dark Radiation, it will be discussed below in section 2. The remaining two ingredients, $W_{ \pm}^{\prime}$ and $M_{\mathrm{mg} \pm}^{\prime}$ are the two Dark Matter candidates in our model, they will be analysed in section 3. We will show that the cosmological production of magnetic monopoles in the Dark sector is enhanced when the $\mathrm{SU}(2)_{D}$ phase transition is of the second order. Consequently, the monopoles contribution to the observed Dark Matter relic density can be sizeable in models with a second order phase transition in the Dark sector. (For the models where the phase transition is strongly first order, it is unlikely.) In section 4 we will combine all three dark ingredients and analyse the effect of the long-range forces acting on the Dark electromagnetic matter and compere with observations.

We will show that both dark monopoles and dark vector bosons are viable dark matter components. The dark matter is not collisionless and hence we will check that it satisfies the known observational constraints. We will further argue that both monopole and vector components of dark matter give the transfer cross-sections of the right magnitude to be able to aid in solving the 'too-big-to-fail' and the 'core-vs-cusp' problems at dwarf galaxy scales.

A viable framework for a fundamental particle theory beyond the Standard Model should address all the sub-Planckian shortcomings of the Standard Model. This includes the generation of: baryon asymmetry, dark matter, and the electroweak scale (together with the stabilisation of the Higgs potential). The origin of neutrino masses, and possibly the solution of strong CP problem and a particle mechanism of cosmological inflation are also on the list. A framework of BSM model building based on classical scale invariance has become popular in recent literature [19-43] as it can address many of these problems in predictive models with small numbers of parameters. The high degree of predictivity/falsifiability arises from the fact that all mass scales have to be generated dynamically and, one cannot

\footnotetext{
${ }^{2}$ In this scenario the induced SM Higgs mass parameter in (1.4) is $\mu_{\mathrm{SM}}^{2}=\lambda_{\mathrm{CWH}}\left\langle\left|\varphi_{\mathrm{CW}}\right|\right\rangle^{2}+\lambda_{\mathrm{P}}\langle|\Phi|\rangle^{2}$.
} 
attempt to extend or repair the model by introducing new mass thresholds where new physics might enter. The approach to self-interacting dark matter in the present paper is consistent with classical scale invariance (even though it is not required). In this case the origin of the dark matter scale (including the monopole mass and the vector boson mass) is tied to the SM electroweak scale and to all other relevant scales of the full model.

Some additional applications of monopoles to dark matter physics were discussed in [44] where $\mathrm{TeV}$-scale monopoles in a hidden sector gave a decaying dark matter candidate due to a small kinetic mixing and a hidden photon mass. In our settings there are no heavy messenger fields between the two sectors to induce the kinetic mixing and the monopoles are stable. In ref. [45] it was pointed out that there is region of parameters in supersymmetric models where invisible monopoles can be the dark matter. On the opposite side of the spectrum, ref. [46] considered galaxy-sized 't Hooft-Polyakov magnetic monopoles.

\section{Dark radiation and $\mathrm{N}_{\text {eff }}$}

The massless dark photon $\gamma^{\prime}$ that remains after the breaking of $\mathrm{SU}(2)_{D}$ to $\mathrm{U}(1)_{D}$ is a new relativistic particle. In this section we will determine the contribution of $\gamma^{\prime}$ to the effective number of relativistic degrees of freedom and apply experimental constraints.

During both BBN and CMB the evolution of the Universe depends on the density of relativistic particles,

$$
\rho_{\text {rel }}=g_{\star}(T) \times \frac{\pi^{2}}{30} T^{4},
$$

and $g_{\star}$ counts the number of all relativistic degrees of freedom. Following standard notation, see e.g. ref. [47] for more detail, $g_{\star}$ is given by,

$$
g_{\star}(T)=\sum_{m_{i}<T} C_{i} g_{i} \times\left(\frac{T_{i}}{T}\right)^{4},
$$

where the sum is over all degrees of freedom, $T_{i}$ and $m_{i}$ are the temperature and the mass of particle $i$, the coefficients are $C_{i}=1$ for bosons and $C_{i}=7 / 8$ for fermions, and $g_{i}$ denotes internal degrees of freedom (e.g. for SM photons $g_{\gamma}=2$ counting two transverse polarisations, and for each flavour of SM neutrino $g_{\nu}=2$ ). This expression is conventionally rewritten in terms of the effective number of neutrinos, $N_{\mathrm{eff}}$ :

$$
\begin{aligned}
g_{\star} & =g_{\gamma}+\frac{7}{8} g_{\nu} N_{\text {eff }} \times\left(\frac{T_{\nu}}{T}\right)^{4}=2+\frac{7}{8} 2 N_{\text {eff }}\left(\frac{4}{11}\right)^{\frac{4}{3}} \\
\Delta N_{\text {eff }} & \simeq 2.2 \Delta g_{\star} .
\end{aligned}
$$

In the Standard Model $N_{\text {eff }}=3.046$ and any new relativistic particles would further add to it. Recently the Planck Collaboration found $N_{\text {eff }}=3.30 \pm 0.27$ at the time of recombination from a combination of CMB and Baryon Acoustic Oscillation data [48]. The projected Planck limit is $\Delta N_{\text {eff }}=0.044$. There is also a limit of $N_{\text {eff }}$ from Helium abundance at BBN $(T=1 \mathrm{MeV}) N_{\text {eff }}=3.24 \pm 1.2(95 \%)$. 
The $\gamma^{\prime}$ is a relativistic particle and contributes to $N_{\text {eff }}$. If the dark photon was in thermal equilibrium with the SM photon, $T_{\gamma^{\prime}}=T$, then eq. (2.2) would give $\Delta g_{\star}=2$ leading to $\Delta N_{\text {eff }} \simeq 4.4$, which is ruled out by the Planck data.

However, this is not what happens in our case where the SM and the hidden Dark sector have no direct mediators and interact only via the Higgs portal; the two sectors will loose thermal contact after the $\mathrm{SU}(2)_{D}$ phase transition to the broken phase and before $\mathrm{BBN}$. The interactions between dark photons and the SM will have to proceed through $\gamma^{\prime}$ coupled to virtual $W^{\prime}$ bosons which are coupled to virtual scalars $\phi$ which have a small mixing with the SM Higgs through the Higgs portal coupling. This interaction rate will be negligible with respect to the Hubble constant, $\Gamma<H=T^{2} / M_{\mathrm{Pl}}^{\star}$, and the hidden sector will be colder than the SM.

Following [47] we will model this situation in terms of two sectors that have had the same temperature when all the degrees of freedom where relativistic, and then decoupled at temperature $T_{D}$. At the time of the measurement, the temperature is $T_{M}$ which is either at recombination or BBN. Assuming that entropy is conserved within each sector we have,

$$
\frac{g_{\star s}^{h}\left(T_{M}^{h}\right) T_{M}^{h 3}}{g_{\star s}^{h}\left(T_{D}\right) T_{D}^{3}}=\frac{g_{\star s}^{\mathrm{sm}}\left(T_{M}\right) T_{M}^{3}}{g_{\star s}^{\mathrm{sm}}\left(T_{D}\right) T_{D}^{3}},
$$

where the superscript $h$ refers to the hidden sector and $s m$ is the Standard Model. The number of relativistic degrees of freedom $g_{\star s}$ relevant for the entropy count is given by the expression (cf. eq. (2.2)),

$$
g_{\star s}(T)=\sum_{m_{i}<T} C_{i} g_{i} \times\left(\frac{T_{i}}{T}\right)^{3} .
$$

In the hidden sector $g_{\star s}^{h}$ counts only $\gamma^{\prime}$ plus relativistic particles that will decay into $\gamma^{\prime}$. Hence,

$$
g_{\star s}^{h}\left(T_{D}\right)=2+n \quad \text { and } \quad g_{\star s}^{h}\left(T_{\mathrm{BBN}}^{h}\right)=g_{\star s}^{h}\left(T_{\mathrm{CMB}}^{h}\right)=2,
$$

where $n$ denotes the number of relativistic particles in the hidden sector, in addition to 2 polarisations of $\gamma^{\prime}$, at the time when the two sectors decouple (i.e. before the phase transition to the broken phase). The number of SM degrees of freedom at the decoupling temperature is

$$
g_{\star s}^{\mathrm{sm}}\left(T_{D}\right)=106.75,
$$

and at the time of measurements,

$$
\begin{aligned}
& g_{\star s}^{\mathrm{sm}}\left(T_{\mathrm{BBN}}\right)=2_{\gamma}+\frac{7}{8}\left(4_{e^{ \pm}}+(3 \times 2)_{\nu}\right)=10.75, \\
& g_{\star s}^{\mathrm{sm}}\left(T_{\mathrm{CMB}}\right)=2_{\gamma}+\frac{7}{8}(3.046 \times 2)_{\nu} \times \frac{4}{11}=3.94 .
\end{aligned}
$$

From eqs. (2.4), (2.2), (2.5) we deduce $\Delta N_{\text {eff }}$ at the time of measurement (BBN or $\mathrm{CMB}$,

$$
\Delta N_{\mathrm{eff}}\left(T_{M}\right)=2.2 \Delta g_{\star}\left(T_{M}\right)=2.2 \times g_{\gamma^{\prime}} \times\left(\frac{T_{M}^{h}}{T_{M}}\right)^{4}=4.4 \times\left(\frac{g_{\star s}^{h}\left(T_{D}\right)}{g_{\star s}^{h}\left(T_{M}^{h}\right)} \frac{g_{\star s}^{\mathrm{sm}}\left(T_{M}\right)}{g_{\star s}^{\mathrm{sm}}\left(T_{D}\right)}\right)^{4 / 3},
$$


so that,

$$
\begin{aligned}
\Delta N_{\text {eff }}\left(T_{\mathrm{CMB}}\right) & =0.022 \times(2+n)^{4 / 3} \\
\Delta N_{\mathrm{eff}}\left(T_{\mathrm{BBN}}\right) & =0.08 \times(2+n)^{4 / 3} .
\end{aligned}
$$

In the model with only the dark photon in the hidden sector we would have $n=0$, leading to $\Delta N_{\text {eff }}\left(T_{\mathrm{CMB}}\right)=0.05$ and $\Delta N_{\mathrm{eff}}\left(T_{\mathrm{BBN}}\right)=0.2$ very similarly to the result in [49].

We can now get a limit on the number $n$ of degrees of freedom in the Dark sector which annihilate into $\gamma^{\prime} .^{3}$ Since the neutral scalar $\phi$ does not couple to the dark photon, the lowest value of $n$ we can have in the Georgi-Glashow Dark sector is $n=6$ given by the three polarisations of $W^{\prime \pm}$. Additional matter fields or higher rank gauge groups would increase $n$ appropriately.

From the Planck limit $\Delta N_{\text {eff }}<0.8(2 \sigma)$ at $T_{\mathrm{CMB}}$ we get an upper limit $n<14(2 \sigma)$. A stronger limit $n \lesssim 7$ follows from the data on Helium abundance at BBN. We conclude that our $\mathrm{SU}(2)_{D}$ gauge theory with an adjoint scalar is consistent with the current available constraints on $N_{\text {eff }}$. At the same time additional degrees of freedom in the Dark sector are disfavoured.

For the minimal case, $n=6$ arising from $W_{ \pm}^{\prime}$ contributions (and assuming that their entropy does not leak to the SM particles ${ }^{4}$ ) our model predicts

$$
\Delta N_{\mathrm{eff}}\left(T_{\mathrm{CMB}}\right)=0.022 \times(2+6)^{4 / 3}=0.35
$$

which could be ruled out by Planck measurements as the projected sensitivity in $\Delta N_{\text {eff }}$ is 0.044 .

\section{Dark matter relic density}

In our model there are two Dark Matter candidates. The massive gauge bosons $W_{ \pm}^{\prime}$ are carriers of the (dark) electric charge of the unbroken $\mathrm{U}(1)_{D}$ and as such are stable. They provide a vector dark matter (VDM) candidate. The dark magnetic (anti)-monopoles $M_{\mathrm{mg} \pm}^{\prime}$ carry topological magnetic charge of $\mathrm{U}(1)_{D}$ and serve as a candidate for the monopole dark matter (MDM). The combined contribution of VDM and MDM should amount to (or not exceed) the observed total dark matter abundance $\Omega_{\mathrm{DM}} h^{2}=0.1187 \pm 0.0017$ measured by the Planck Satellite [48].

\subsection{Dark gauge bosons: Sommerfeld enhancement and relic density}

$W_{+}^{\prime}$ and $W_{-}^{\prime}$ can annihilate into two dark photons $\gamma^{\prime}$ or into two $\phi$ scalars. The dominant contribution to their annihilation is given by the Feynman diagrams in figure 1. Using these

\footnotetext{
${ }^{3}$ Even if some of these particles have a relic density of the right order of magnitude to give the correct dark matter density, almost all of the entropy in the species will have been transferred since freeze out normally happens at $T=M / 20$. The vector bosons $W_{ \pm}^{\prime}$ can decay to both $\gamma^{\prime}$ and $\phi$. Since $\phi$ mixes with the SM Higgs, this entropy will leak to the Standard Model particles, which could effectively increase $g_{\star s}^{\mathrm{sm}}\left(T_{D}\right)$. The fraction of the entropy transferred to $\gamma^{\prime}$ is given by the branching ratio $\Gamma_{W_{+}^{\prime} \rightarrow \gamma^{\prime}}$ which is assumed to dominate over the entropy transfer to the SM.

${ }^{4}$ Since $\phi$ mixes with the SM Higgs there is some entropy exchange between the two sectors which can increase $g_{\star s}^{\mathrm{sm}}\left(T_{\mathrm{CMB}}\right)$.
} 

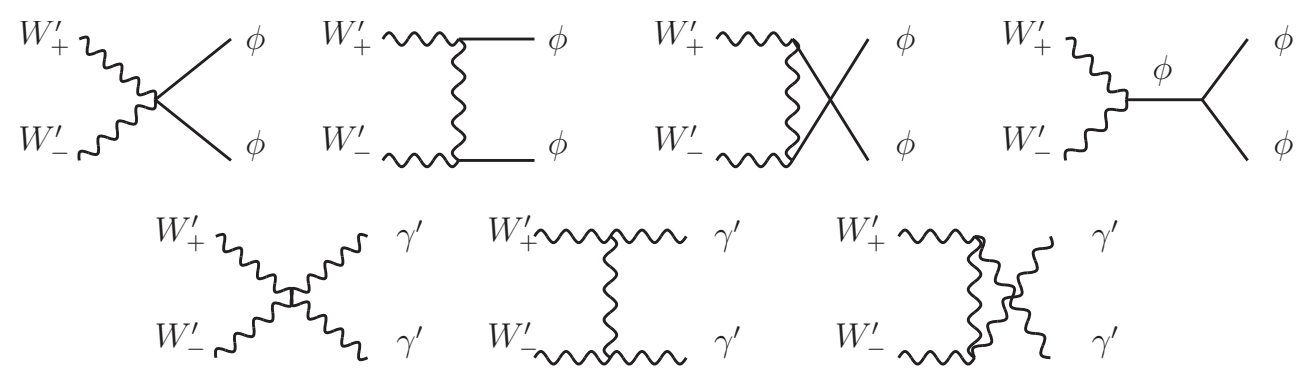

Figure 1. Diagrams giving dominant contribution to the $W_{ \pm}^{\prime}$ annihilation cross-section.

we have computed the leading order non-relativistic s-wave annihilation cross-section, ${ }^{5}$

$$
\langle\sigma v\rangle_{\text {pert }}=\frac{1579 g_{D}^{4}}{2304 \pi M_{W^{\prime}}^{2}}-\frac{5 g_{D}^{2} \lambda_{\phi}}{192 \pi M_{W^{\prime}}^{2}}+\frac{3 \lambda_{\phi}^{2}}{64 \pi M_{W^{\prime}}^{2}} .
$$

This leading order perturbative cross-section is further enhanced at low velocities by the Sommerfeld effect [50-54], which arises from multiple dark photon exchanges in the $t$ channel between the incoming $W_{+}^{\prime}$ and $W_{-}^{\prime}$. As the result we have,

$$
\langle\sigma v\rangle=S\langle\sigma v\rangle_{\text {pert }}
$$

where the multiplicative Sommerfeld factor $[50,54]$ is

$$
S=\frac{\alpha_{D} \pi}{v} \frac{1}{1-\exp \left[-\frac{\alpha_{D} \pi}{v}\right]},
$$

and becomes relevant in the non-relativistic regime where the 'perturbative' factor $\frac{\alpha_{D} \pi}{v}$ is no longer small.

The relic density of vector dark matter is found by solving the Boltzmann equations,

$$
\frac{d n_{i}}{d t}+3 H n_{i}=-\langle\sigma v\rangle\left(n_{i}^{2}-n_{i}^{e q 2}\right),
$$

where $n_{i}$ for $i=1,2$ is the density of $W_{+}^{\prime}$ and $W_{-}^{\prime}$ with $n_{1}=n_{2}$. Then the combined $W_{ \pm}^{\prime}$ density $n$ is twice that, $n=2 n_{1}=2 n_{2}$. It satisfies the equation

$$
\frac{d n}{d t}+3 H n=-\langle\sigma v\rangle_{\mathrm{eff}}\left(n^{2}-n^{e q 2}\right), \quad \text { where } \quad\langle\sigma v\rangle_{\mathrm{eff}}:=\frac{\langle\sigma v\rangle}{2} .
$$

Using this Boltzmann equation we can now write down the standard s-wave solution for the dark matter abundance, see e.g. [55, 56],

$$
\Omega_{\mathrm{VDM}} h^{2}=1.07 \times 10^{9} \frac{x_{f} \mathrm{GeV}^{-1}}{\left(g_{\star s} / \sqrt{g_{\star}}\right) M_{\mathrm{Pl}}\langle\sigma v\rangle_{\mathrm{eff}}},
$$

\footnotetext{
${ }^{5}$ For simplicity, in the analytic expression on the r.h.s. of (3.1) we have assumed that $m_{\phi} \ll M_{W^{\prime}}$. We have checked that the inclusion of effects due to scalar masses does not make a noticeable change in our numerical results.
} 


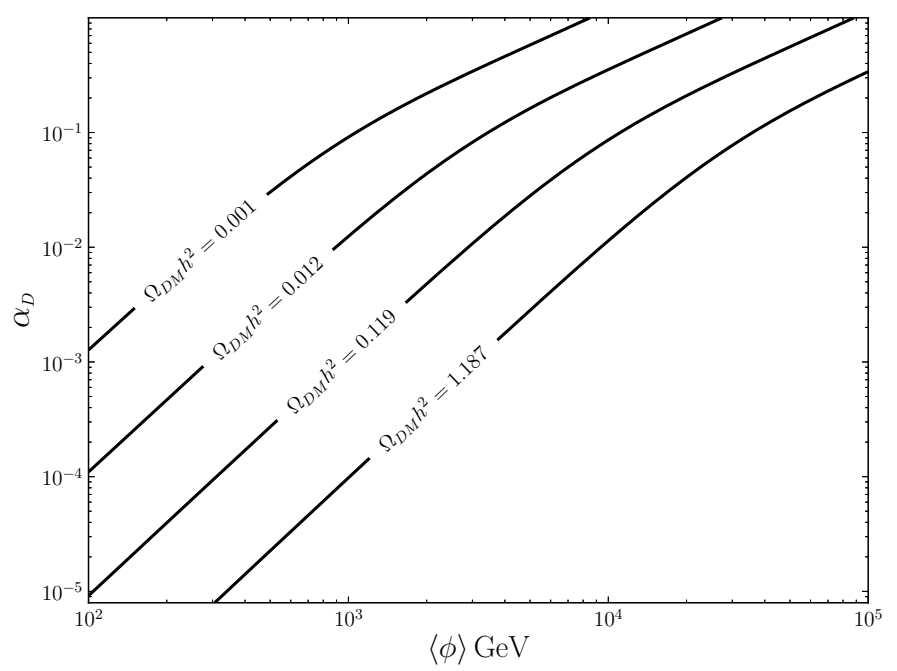

Figure 2. Contours of the relic density of Vector Dark Matter.

where $x_{f}:=M_{W^{\prime}} / T_{f}$ and $T_{f}$ is the freeze-out temperature. The expression for $x_{f}$ is

$$
x_{f}=\log \left(0.038 \frac{g}{\sqrt{g_{\star}}} M_{\mathrm{Pl}} M_{W^{\prime}}\langle\sigma v\rangle_{\mathrm{eff}}\right)-\frac{1}{2} \log \log \left(0.038 \frac{g}{\sqrt{g_{\star}}} M_{\mathrm{Pl}} M_{W^{\prime}}\langle\sigma v\rangle_{\mathrm{eff}}\right),
$$

where $g=6$ is the number of $W_{ \pm}^{\prime}$ degrees of freedom.

The relic density of $W_{ \pm}^{\prime}$ given by eqs. (3.6)-(3.7) is shown in figure 2 on the twodimensional plane $\left(\alpha_{D}, w\right)$ of the Dark sector parameter space. In the CW case $\lambda_{\phi} \ll \alpha_{D}$ and the scalar self-coupling $\lambda_{\phi}$ plays no role. We have also considered a more general case with $\lambda_{\phi} / \alpha_{D}=$ fixed, for example $=4$ similarly to the SM value, and continued to scan over $\alpha_{D}$ and $\langle\phi\rangle$. We have found no noticeable difference in the relic density behaviour in figure 2 .

The relic density curves in figure 2 are seen to be bending at higher values of $\alpha_{D}$. This is the consequence of the Sommerfeld enhancement factor in (3.3). Indeed, while $S=1$ in the perturbative regime $\frac{\alpha_{D} \pi}{v} \ll 1$, its behaviour changes to $S=\frac{\alpha_{D} \pi}{v}$ in the regime of larger gauge coupling or equivalently lower velocities — which is precisely the reason for the bending. The velocity estimate is $v=\sqrt{T_{f} / M_{W^{\prime}}}=1 / \sqrt{x_{f}}$. In scanning over the parameter space in figure 2 we found $x_{f}$ changing between 15 and 25 which gave the range of velocities $0.2 \lesssim v \lesssim 0.25$ in the Sommerfeld $S$ factor.

\subsection{Dark monopoles}

\subsubsection{Production of monopoles}

Monopoles are topological defects which are produced during the phase transition in the early Universe. First we need to determine the order of the phase transition of the $\mathrm{SU}(2)_{D}$ dark sector relevant for the monopole production. At sufficiently high temperature the only minimum of the effective potential of the Dark sector $V_{D}(\phi, T)$ is at the origin $\phi=0$ (here $\phi$ is the Dark sector scalar in the unitary gauge) and the $\mathrm{SU}(2)_{D}$ is restored. As the 
Universe expands, the second minimum appears, and at critical temperature, $T=T_{c}$, the values of $V_{D}$ in the two minima become equal. The phase transition is of the first order if there is a barrier separating the two minima at critical temperature. If, on the other hand, there is no potential barrier between the minima, the phase transition is of the second order.

As already noted in section 1.3 the character of the phase transition depends on whether the Dark sector is of the CW-type or is distinct from it. A simple estimate suffices to illustrate this point; to this end we proceed by writing the one-loop thermal potential in the form $[16,17]$

$$
V_{D}(\phi, T)=D\left(T^{2}-T_{0}^{2}\right) \phi^{2}-E T \phi^{3}+\frac{\lambda_{T}}{4} \phi^{4},
$$

with the parameters in our case (i.e. the model of (1.1)) given by,

$$
D=\frac{g_{D}^{2}}{4}, \quad E=\frac{g_{D}^{3}}{2 \pi}, \quad T_{0}=\frac{1}{4 D}\left(\sqrt{2} m^{2}-\frac{g_{D}^{4}}{2 \pi} w^{2}\right), \quad \lambda_{T}=\lambda_{\phi}-\frac{3 g_{D}^{4}}{8 \pi^{2}} \log \frac{g_{D}^{2} w^{2}}{a_{B} T^{2}}
$$

and $a_{B} \simeq e^{3.91}$. We get the critical temperature, $T_{c}$, when the values of $V_{D}$ in the two minima become equal,

$$
T_{c}^{2}=\frac{T_{0}^{2}}{1-E^{2} /\left(\lambda_{T} D\right)}, \quad \phi_{c}=\frac{2 E T_{c}}{\lambda_{T_{c}}},
$$

with $\phi_{c}$ being the value of the field in the second minimum at this instance. The strength of the first order phase transition is conventionally characterised by the dimensionless order parameter $\phi_{c} / T_{c}$, which can be thought of as the separation between the two vacua in units of temperature. We have

$$
\frac{\phi_{c}}{T_{c}}=\frac{2 E}{\lambda_{T_{c}}}=\frac{g_{D}^{3}}{\pi\left(\lambda_{\phi}+\frac{3 g_{D}^{4} \times 3.91}{8 \pi^{2}}\right)} .
$$

Strongly first order phase transitions have $\phi_{c} / T_{c} \gtrsim 1$. The phase transition is weakly first order if the vacua at 0 and $\phi_{c}$ are near each other, and the phase transition changes from the very weakly first order to the second order for $\phi_{c} / T_{c} \ll 1$.

To have a second order phase transition we need:

$$
\frac{g_{D}^{3}}{\pi} \ll \lambda_{\phi}+\frac{3 g_{D}^{4} \times 3.91}{8 \pi^{2}} .
$$

In the Coleman-Weinberg settings $\lambda_{\phi}=\frac{11 g_{D}^{4}}{8 \pi^{2}}$, which implies

$$
\mathrm{CW}: \quad \frac{\phi_{c}}{T_{c}}=\frac{1}{g_{D}} \frac{8 \pi}{22.73}=\frac{1}{3.2 \sqrt{\alpha_{D}}} \gg 1 \quad \text { for } \quad \alpha_{D} \ll 0.1,
$$

which gives the strongly first order phase transition for the weakly coupled CW sector, as expected. 
The production of magnetic monopoles in early Universe can be estimated using the Kibble limit [14]. It is a lower limit on the density of magnetic monopoles created cosmologically, it is expressed in terms of the horizon volume, and it applies to both, 1st and 2nd order phase transitions,

$$
\frac{n_{\mathrm{m}}}{T^{3}} \geq\left(\frac{T_{c}}{\sqrt{\frac{45}{4 \pi^{3} g_{\star}} M_{\mathrm{Pl}}}}\right)^{3} .
$$

First we justify this bound for the phase transition of the 2nd order [12, 14]. During the phase transition the $\phi_{a}$ field changes from 0 to $|\phi|^{2}=w^{2}$. The direction of $\phi_{a}$ is the same inside a volume, $\zeta^{3}$, where $\zeta$ is the correlation length. At the critical temperature $\zeta$ diverges, but due to causality, information can only be exchanged inside the horizon. The correlation length will be frozen in at the horizon scale $d_{h} \simeq H^{-1}$ and we will get a domain structure, with $\phi_{a}$ in different domains pointing in different directions. At domain intersection points the random orientation of the scalar field, given a non-trivial topology, can give rise to magnetic monopoles with a probability $p$ close to 1 . We can estimate the density of monopoles created [12]:

$$
n_{\mathrm{m}} \propto p \zeta^{-3} \sim \zeta^{-3}, \quad \text { where } \quad \zeta<d_{h}=H^{-1},
$$

and equation (3.14) follows.

If the phase transition in the Dark sector was of the first-order, a potential barrier is formed between the symmetric and the symmetry breaking vacua, and below the critical temperature, the symmetric vacuum is meta-stable. Bubbles of the symmetry breaking vacuum will nucleate and expand. Inside each bubble the scalar field will have one random orientation. When the bubbles collide they can create magnetic monopoles. The density of magnetic monopoles will therefore proportional to the density of bubbles. Since the bubbles can not propagate faster than the speed of light the size of a bubble is limited by the horizon size. We therefore get a very similar bound [56] on the density of magnetic monopoles as from the Kibble argument in eq. (3.14), enhanced by a logarithmic factor [57],

$$
\text { 1st order ph.tr. : } \quad \frac{n_{\mathrm{m}}}{T^{3}} \geq\left[\frac{T_{c}}{\sqrt{\frac{45}{4 \pi^{3} g_{\star}}} M_{\mathrm{Pl}}} \log \left(\frac{\sqrt{\frac{45}{4 \pi^{3} g_{\star}}} M_{\mathrm{Pl}}}{T_{c}}\right)^{4}\right]^{3} \text {. }
$$

Importantly, in the case of second-order phase transitions, the Kibble bound was refined by Zurek [15] with an argument relying on a careful analysis of the timescales involved. The system undergoing the phase transition is characterised by the relaxation time $\tau$, and the correlation length $\zeta$,

$$
\tau=\frac{\tau_{0}}{\sqrt{|\epsilon(T)|}} \quad \text { and } \quad \zeta=\zeta_{0}|\epsilon(T)|^{-\nu},
$$

where

$$
\epsilon(T):=\frac{T-T_{c}}{T_{c}},
$$


and $1 / 2$ and $\nu$ are the critical exponents describing the degree of divergence of $\tau$ and $\zeta$ in the proximity of the critical temperature $T_{c}$. At the time $t$ close to the critical point $t_{c}$ one has $t-t_{c} \propto \epsilon(T) \rightarrow 0$ where the proportionality constant is the quenching time-scale,

$$
\tau_{Q}:=\frac{t-t_{c}}{\epsilon(T)} .
$$

At the time $t_{\star}$ when the time interval to the critical point becomes equal to the relaxation time $\tau$, the system is no longer able to re-adjust. At this instance we have,

$$
\left|t_{\star}-t_{c}\right|=\tau\left(t_{\star}\right)=\tau_{0}\left|\epsilon\left(t_{\star}\right)\right|^{-1 / 2},
$$

with the 1.h.s. being via (3.19) also $=\tau_{Q}\left|\epsilon\left(t_{\star}\right)\right|$, which implies that

$$
\left|\epsilon\left(t_{\star}\right)\right|^{3 / 2}=\tau_{0} / \tau_{Q} \quad \text { and } \quad \zeta\left(t_{\star}\right)=\zeta_{0}\left|\epsilon\left(t_{\star}\right)\right|^{-\nu}=\zeta_{0}\left|\tau_{0} / \tau_{Q}\right|^{2 \nu / 3} \text {. }
$$

In our case $\tau_{Q}=H\left(T_{c}\right)^{-1}$ and for the remaining constants, from the Landau-Ginzburg theory one estimates [6] that $\zeta_{0} \simeq \tau_{0} \sim 1 /\left(\sqrt{\lambda_{\phi}} T_{c}\right)$. Classical value for the critical exponent $\nu$ is $1 / 2$ but quantum corrections can modify this value.

The second equation in (3.21) is the correlation length at the freeze-out temperature $t_{\star}$, it is the more accurate replacement of the Kibble-limit estimate $\zeta<d_{h}=H\left(T_{c}\right)^{-1}$.

The monopole relic density from the Zurek mechanism today is then given by the following expression $[6,15]$ (for conversion factors see eqs. (3.42)-(3.43)),

$$
\text { 2nd order ph.tr. : } \quad \frac{n_{\mathrm{m}}}{T^{3}} \simeq 10^{-2}\left(\frac{M_{\mathrm{m}}}{1 \mathrm{TeV}}\right)\left(\frac{30 T_{c}}{M_{\mathrm{Pl}}}\right)^{\frac{3 \nu}{1+\nu}},
$$

or (using numerical conversion eqs. (3.42)-(3.43)),

$$
\text { 2nd order ph.tr. : } \quad \Omega_{\mathrm{m}} h^{2}=1.5 \times 10^{9}\left(\frac{M_{\mathrm{m}}}{1 \mathrm{TeV}}\right)\left(\frac{30 T_{c}}{M_{\mathrm{Pl}}}\right)^{\frac{3 \nu}{1+\nu}} .
$$

Zurek's construction above is also valid for various condensed matter systems where the effect has been experimentally confirmed [58, 59].

The main difference between the Zurek result (3.22)-(3.23) and the Kibble lower limit (3.14) or (3.16), is the power $p$ of the $\left(T_{c} / M_{\mathrm{Pl}}\right)^{p}$ suppression factor. It reduces from $p=3$ in the Kibble bound to the $p=3 \nu /(1+\nu) \simeq p_{\mathrm{cl}}=1$ for $\nu_{\mathrm{cl}}=1 / 2$ in the Zurek bound. This makes it possible for relatively light monopoles with masses starting in a few hundred $\mathrm{TeV}$ range to contribute to dark matter, as can be inferred from figure 4 in section 3.2.2. The Kibble bound would require monopoles to be at least in the $10^{11} \mathrm{GeV}$ range or above to play a non-negligible role in the dark matter relic abundance, cf. figure 3.

\subsubsection{Evolution of monopoles}

Magnetic monopoles are stable and can not decay due to conservation of their dark magnetic charge. The density of magnetic monopoles, once created, can therefore only be changed by monopole-anti-monopole annihilation. 


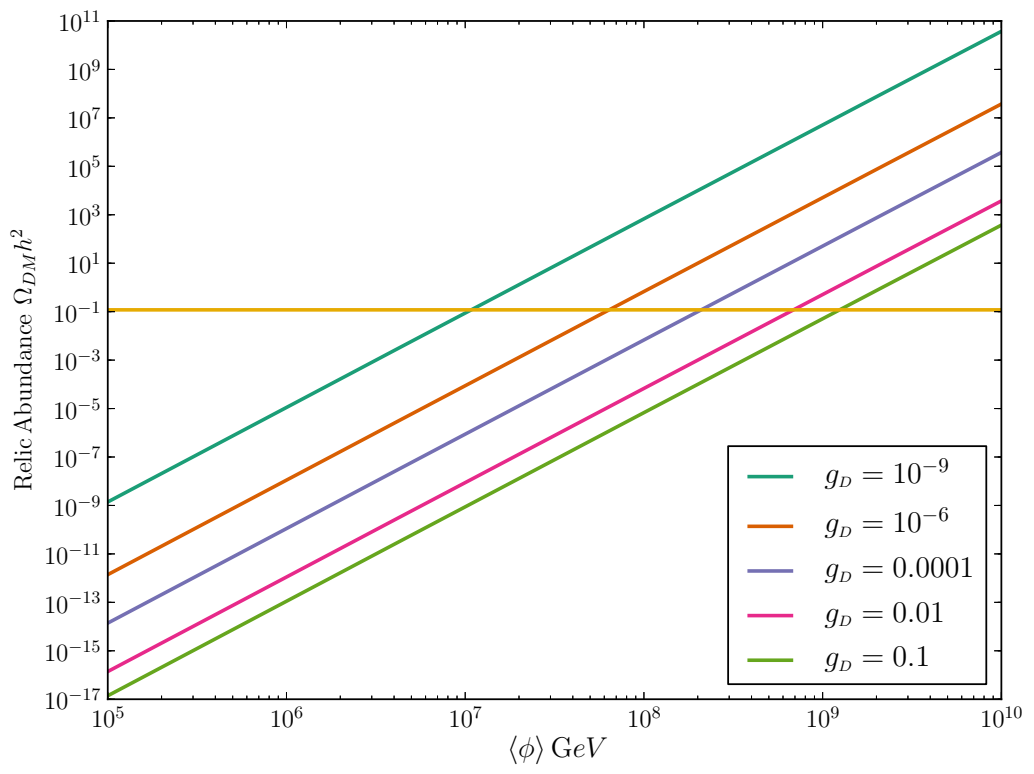

Figure 3. The relic density of monopoles produced cosmologically during a first order phase transition as a function of the dark scalar vev $w=\langle\phi\rangle$ and for different values of the dark gauge coupling $g_{D}$.

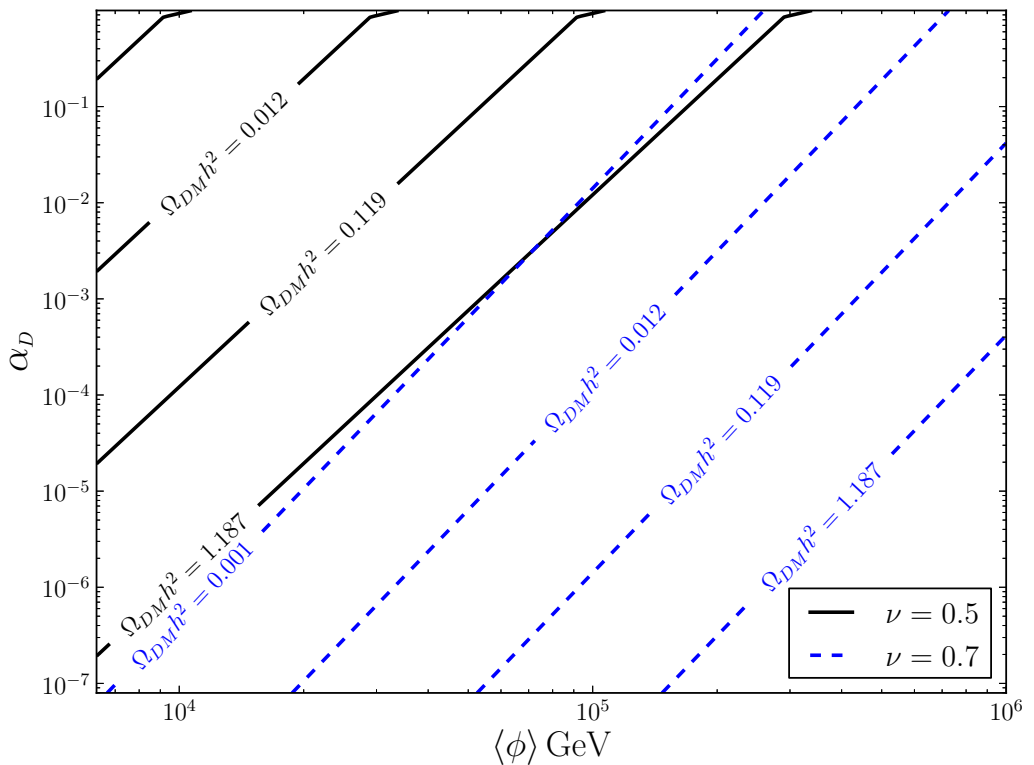

Figure 4. The relic density of monopoles after a second order phase transition. Results are shown on the Dark sector gauge-coupling-vev plane for two different values for the critical exponent, $\nu=0.5$ (in black) and $\nu=0.7$ (in blue). 
In the diffusion approach [60-62], the motion of monopoles in a plasma of electrically charged particles, in our case $W_{ \pm}^{\prime}$, is described by the Brownian walk with thermal velocities $v_{T}=\sqrt{T / M_{\mathrm{m}}}$ and the mean free path $l_{\text {free }}$,

$$
l_{\text {free }}=v_{T} t_{\text {free }}=\sqrt{\frac{T}{M_{\mathrm{m}}}} \frac{M_{\mathrm{m}}}{T \sum_{i} n_{i} \sigma_{i}},
$$

where $\sigma_{i}$ is the classical cross-section for large angle scattering of a light particle with a monopole,

$$
\sigma_{i}=\frac{g_{\mathrm{mD}}^{2} q_{i}^{2}}{(4 \pi)^{2} T^{2}}
$$

$n_{i}$ is the number density and the sum is over all spin states. The number density for relativistic particles is [56]:

$$
n_{i}=\frac{\zeta(3)}{\pi^{2}} T^{3}
$$

and for non relativistic particles of mass $M_{i}$ the number density is

$$
n_{i}=\left(\frac{M_{i} T}{2 \pi}\right)^{\frac{3}{2}} \exp \left(-\frac{M_{i}}{T}\right) .
$$

It is convenient to define the dimensionless quantity $B$,

$$
B:=T^{-1} \sum_{i} n_{i} \sigma_{i}, \quad \text { so that }: \quad l_{\text {free }}=\frac{1}{B} \sqrt{\frac{T}{M_{\mathrm{m}}}} \frac{M_{\mathrm{m}}}{T^{2}} .
$$

The attractive Coulomb force between the monopoles and anti-monopoles makes them drift towards each other during their random walk in the electric plasma. Their drift velocity is determined from the balance between the monopole-anti-monopole attraction and the drag force of the plasma. It is given by [62],

$$
v_{\text {drift }}(r)=\frac{1}{B} \frac{g_{\mathrm{mD}}^{2}}{T^{2} r^{2}}
$$

Monopoles drift toward anti-monopoles through the plasma, the drag force dissipates monopole energy, and if the mean free path is less than the capture radius,

$$
l_{\text {free }} \leq l_{\text {capt }}=g_{\mathrm{mD}}^{2} /(4 \pi T),
$$

the monopole-anti-monopole bound state is formed which ultimately annihilates to the ordinary elementary states. The relevant time scale for the formation of the bound state is $t_{\mathrm{drift}}=r / v_{\mathrm{drift}}=1 / \Gamma_{\mathrm{drift}}$. Therefore, the monopole-anti-monopole annihilation crosssection is given by,

$$
\sigma=\frac{\Gamma_{\mathrm{drift}}}{n_{\mathrm{m}}}=\frac{v_{\mathrm{drift}}(r)}{n_{\mathrm{m}} r}=\frac{1}{B} \frac{g_{\mathrm{mD}}^{2}}{T^{2}} .
$$

The resulting density of monopoles after annihilation is determined by the Boltzmann equation [61],

$$
\frac{d}{d x} \frac{n_{\mathrm{m}}}{s}=\frac{\sigma}{H(x) x}\left(\frac{n_{\mathrm{m}}}{s}\right)^{2}, \quad \text { where } \quad x:=\frac{M_{\mathrm{m}}}{T}
$$


with $\sigma$ on the right hand side given by (3.31). The solution is known analytically [61], it quickly becomes independent of the initial conditions at $x_{0}$, resulting in,

$$
\frac{n_{\mathrm{m}}}{s}(x) \simeq \frac{2 \pi B}{g_{\mathrm{mD}}^{2}} \frac{\sqrt{\frac{45}{4 \pi^{3} g_{\star}}} M_{\mathrm{m}}}{M_{\mathrm{Pl}}} \frac{1}{x} .
$$

If, following [61], we assume that the the plasma consists of particles that are relativistic from $x_{0}$ to $x_{f}$ where $x_{f}$ corresponds to the temperature where $l_{\text {free }}$ and $l_{\text {capt }}$ become equal,

$$
x_{f}^{-1}=\left(\frac{4 \pi}{g_{\mathrm{mD}}^{2}}\right)^{2} \frac{1}{B^{2}}
$$

the result for the final number density of monopoles is in agreement with [61],

$$
\frac{n_{\mathrm{m}}}{s}\left(x_{f}\right) \simeq \frac{2 \pi}{B g_{\mathrm{mD}}^{2}}\left(\frac{4 \pi}{g_{\mathrm{mD}}}\right)^{2} \frac{\sqrt{\frac{45}{4 \pi^{3} g_{\star}}} M_{\mathrm{m}}}{M_{\mathrm{Pl}}} .
$$

This diffusive capture process is effective only as long as the mean free path is smaller than the capture radius. At lower temperatures, where $l_{\text {free }}$ exceeds $l_{\text {capt }}$, the rate of monopolesanti-monopole annihilation cannot compete with the Universe expansion and the monopole density freezes out at the value at $x_{f}$.

There is an important difference between the more standard application of the diffusion method described above, where GUT monopoles were propagating in the plasma of very light relativistic electrons and positrons, and our model. In our case the plasma is made up of $W_{ \pm}^{\prime}$ with masses $M_{W^{\prime}}=g_{D}\langle\phi\rangle$ much closer to the monopoles of the same dark sector. Thus, the particles in the plasma will become non-relativistic fairly soon after the phase transition, when

$$
x_{\mathrm{nr}}=\frac{M_{\mathrm{m}}}{T_{\mathrm{nr}}}=\frac{M_{\mathrm{m}}}{M_{W^{\prime}}}=\frac{1}{\alpha_{D}}=\frac{4 \pi}{g_{\mathrm{mD}}^{2}} .
$$

After $x_{\mathrm{nr}}$ the density of the plasma will decrease exponentially, as per (3.27), and the mean free path will therefore exponentially increase. The final monopole density in our model will thus be cut-off at $x_{\mathrm{nr}}$

$$
\frac{n_{\mathrm{m}}}{s}\left(x_{\mathrm{nr}}\right) \simeq \frac{B}{2} \frac{\sqrt{\frac{45}{4 \pi^{3} g_{\star}}} M_{m}}{M_{\mathrm{Pl}}} .
$$

\subsubsection{Current density of monopoles}

To determine the current density in monopoles we first have to determine the type of the Dark sector phase transition and compute the initial monopole production density accordingly. If the initial production density is lower than the estimated density after monopole-anti-monopole annihilation (3.37), the effect of annihilations is unimportant and the initial monopole density survives. If on the other hand the initial density is higher than the annihilation density, the final monopole density is set by monopole-anti-monopole annihilations expression. 
The conversion from monopole density, $n_{\mathrm{m}} / s$ or $n_{\mathrm{m}} / T$, to $\Omega_{\mathrm{m}} h^{2}$ is standard,

$$
\begin{aligned}
\Omega_{\mathrm{m}} h^{2} & =\rho_{\mathrm{m}} \frac{1}{\rho_{\text {crit }} h^{-2}}, \\
\rho_{\mathrm{m}} h & =\frac{n_{\mathrm{m}}}{s} M_{\mathrm{m}} s_{0}=\frac{n_{\mathrm{m}}}{T^{3}} M_{\mathrm{m}} T_{0}^{3},
\end{aligned}
$$

where subscript 0 refers to the current time or temperature and the normalisation factors are given by,

$$
\begin{aligned}
\rho_{\text {crit }} h^{-2} & =1.9 \times 10^{-29} \mathrm{gcm}^{-3}=7.53 \times 10^{-47} \mathrm{GeV}^{4}, \\
s_{0} & =\frac{2 \pi^{2}}{45} g_{\star}\left(t=t_{0}\right) T_{0}^{3},
\end{aligned}
$$

with $T_{0}=T_{\mathrm{CMB}}=2.73 \mathrm{~K}=2.35 \times 10^{-13} \mathrm{GeV}$ and $g_{\star}\left(t=t_{0}\right)=2$ in the Dark sector and 3.94 in the SM. Thus

$$
\begin{aligned}
\Omega_{\mathrm{m}} h^{2} & =\frac{n_{\mathrm{m}}}{s} \times \frac{M_{\mathrm{m}}}{1 \mathrm{TeV}} \times 1.5 \times 10^{11}, \\
& =\frac{n_{\mathrm{m}}}{T^{3}} \times \frac{M_{\mathrm{m}}}{1 \mathrm{TeV}} \times 1.7 \times 10^{11} .
\end{aligned}
$$

The current relic density of monopoles for a first order phase transition computed using (3.16), is shown in figure 3. We see that relic density depends strongly on the dark scalar field vev $w=\langle\phi\rangle$ as this sets both the mass of the monopoles and the critical temperature of the phase transition. The density increases with lower coupling $g_{D}$ as the mass of the monopoles increase.

The current relic density for a second order phase transition, based on (3.22)-(3.23) combined with (3.37), is plotted in figure 4 for two values of the critical exponent, $\nu=0.5$ and $\nu=0.7$.

For a second order phase transition we can see that we have two components of dark matter both with a significant fraction of the observed relic density. The combined relic density can be seen in figure 5 for $\nu=0.5$ and in figure 6 for $\nu=0.6$.

Note added. Dark sector monopoles and vector bosons were also considered recently in ref. [7] which concluded that the monopole contribution to the dark matter density is several orders of magnitude smaller than the observed dark matter relic density, and thus should be negligible. This does not agree with our findings in figures 5-6. In computing the relic abundance of magnetic monopoles in the dark sector, the authors of [7] — ArXiv version 1 - appear to have concentrated only on a narrow patch of the available parameter space where both the electric $g_{D}$ and magnetic $g_{\mathrm{mD}}=\frac{4 \pi}{g_{D}}$ coupling constants of the dark sector are not strongly coupled, $g_{\mathrm{mD}} \sim g_{D} \sim 1$. Our analysis, on the other hand, only requires a weakly-coupled electric theory formulation. The 't Hooft-Polyakov monopoles in this regime continue to be well described by the semi-classical theory in this weak electric coupling regime. In the ArXiv version 2 of ref. [7] which has appeared after our paper, the earlier restriction of being in the weak magnetic coupling regime was lifted. However the authors of ref. [7] have still concluded based on their figure 8 that to achieve the 


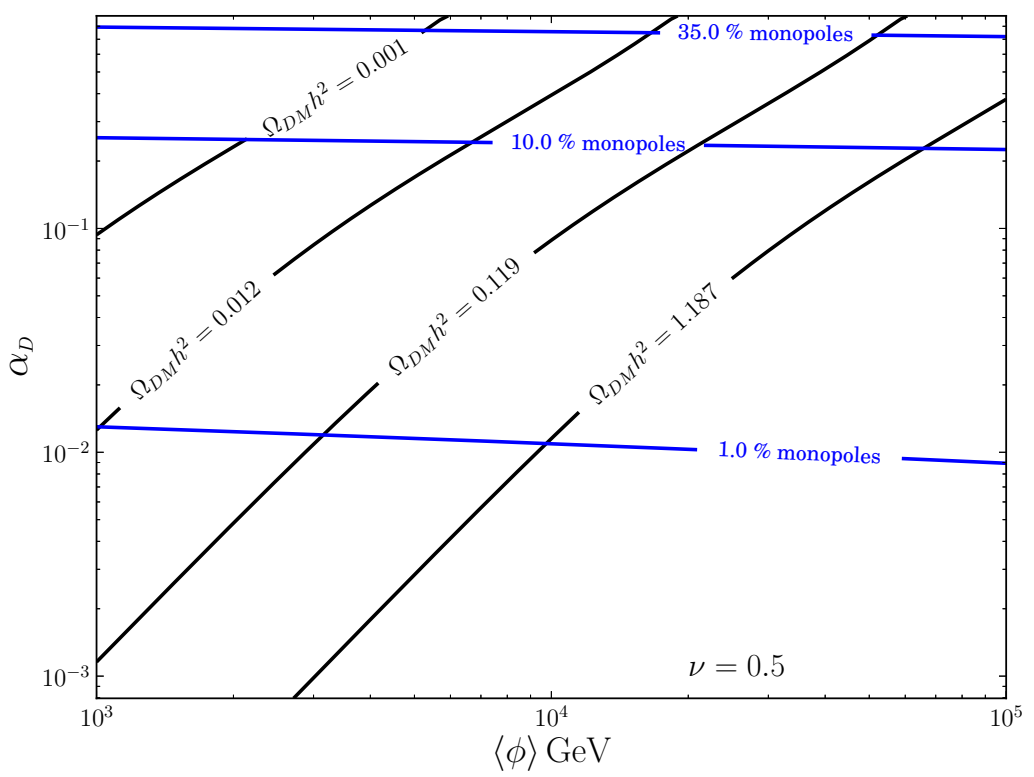

Figure 5. Combined relic density of vector and monopole components of dark matter after a second order phase transition with the critical exponent $\nu=0.5$. The blue lines show the relative fraction of monopoles.

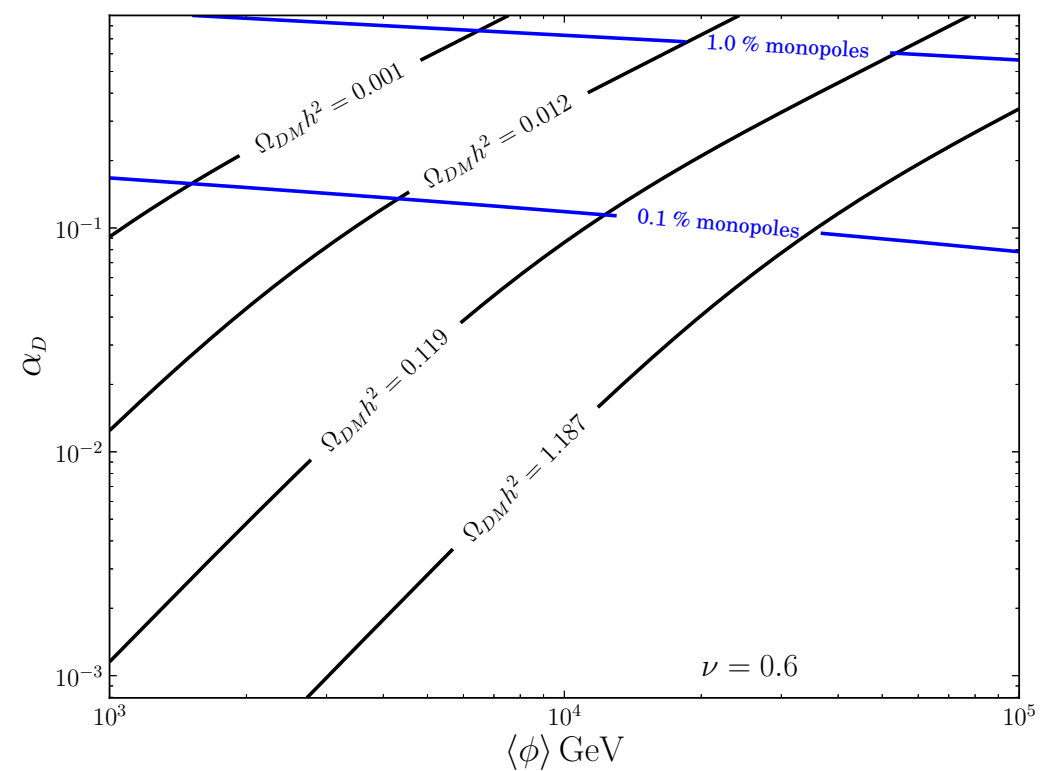

Figure 6. Combined relic density for monopole and vector dark matter with $\nu=0.6$. Blue contours show the fraction of monopoles alone.

monopole abundance of $10 \%$ of the observed dark matter relic density would require what they describe as a $10^{-2} \%$ fine-tuning. Our results in figures 5-6 do not support such a conclusion. It follows from our figure 5 that the contour of the observed relic density value $\Omega_{\mathrm{DM}} h^{2}=0.119$ can readily intersect the $10 \%$ monopole abundance contour,and even the $35 \%$ monopole abundance contour, and so on. 


\section{Self-interacting dark matter}

Due to the unbroken $\mathrm{U}(1)_{D}$ symmetry we will have long-raged forces acting on the dark matter particles. Vector dark matter is electrically charged under the $\mathrm{U}(1)_{D}$ while the magnetic monopoles have magnetic charges. This self-interacting dark matter provides a framework which can solve cosmological problems of collisionless cold dark matter (CCDM) at small scales [63]. Numerical simulations [64] based on CCDM are very successful in describing the large scale structure of the Universe at scales $\gg 1 \mathrm{Mpc}$. However observations on galactic and subgalactic scales $\lesssim 1 \mathrm{Mpc}$ are in conflict with the structure formation predicted by such simulations [64, 65].

Collisionless dark matter predicts that density distributions of dwarf galaxy halos should have a cusp in the centre, while observationally flat cores have been found; this is the core-vs-cusp problem. Cold dark matter simulations also predict too many too large sub-halos in the Milky Way. In particular, simulations which use collisionless dark matter predict $\mathcal{O}(10)$ sub-halos with velocities $v>30 \mathrm{~km} / \mathrm{s}$, but no halos have been observed with $v>25 \mathrm{~km} / \mathrm{s}$. This is known as the 'too-big-to-fail' problem.

In order to address these problems with small scale structure models of self-interacting dark matter have been proposed and studied in recent literature. References [66-71] considered long-range Yukawa interactions between cold dark matter mediated by a light vector or scalar boson. The effects of an unbroken U(1) symmetry with a massless force carrier were considered in [55, 72-74].

The result of self-interactions is to transfer energy between the dark matter particles. This effect is normally captured by the transfer cross-section defined by,

$$
\sigma_{T}=\int d \Omega(1-\cos \theta) \frac{d \sigma}{d \Omega},
$$

where $d \sigma / d \Omega$ is the usual differential cross-section. Even though our model contains a microscopically massless force carrier $\gamma^{\prime}$, in a plasma it is described by the Yukawa potential,

$$
V(r)=\frac{\alpha_{e}}{r} e^{-m_{\gamma^{\prime}} r},
$$

where the effective mass of $\gamma^{\prime}$ is due to its interactions with the plasma and is given by the inverse of the Debye length $l_{\mathrm{D}}$,

$$
m_{\gamma^{\prime}}=\frac{1}{l_{\mathrm{D}}}=\frac{\left(4 \pi \alpha_{D} \rho\right)^{1 / 2}}{M_{\mathrm{DM}} v} .
$$

Here $\rho$ is the dark matter density in a galaxy and $v$ is its velocity. Since the density $\rho$ is small, the effective mass $m_{\gamma^{\prime}}$ will be small and we can use the classical Coulomb limit $M_{\mathrm{DM}} v / m_{\gamma^{\prime}} \gg 1$ for both the attractive and repulsive potential with the result $[70,75,76]$,

$$
\sigma_{T}=\frac{16 \pi \alpha_{D}^{2}}{M_{\mathrm{DM}}^{2} v^{4}} \log \left(1+\frac{M_{\mathrm{DM}}^{2} v^{2}}{2 \alpha_{D} m_{\gamma^{\prime}}^{2}}\right) .
$$

If the energy transfer is large enough, self interacting dark matter could flatten out the cores of dwarf galaxies and decrease the number of large subhaloes, solving the core-vs-cusp 


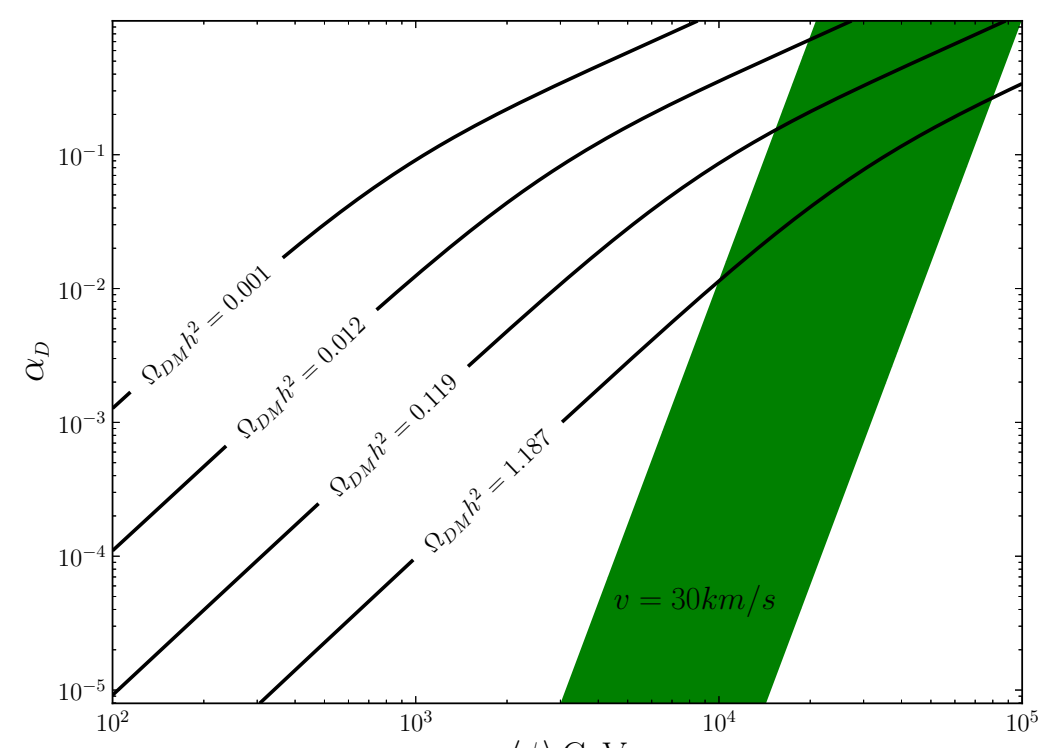

$\langle\phi\rangle \mathrm{GeV}$

Figure 7. Vector Dark Matter transfer cross-section and the relic density. The green region shows the region in parameter space where $\sigma_{T} / m_{\mathrm{DM}}$ is in the interval between 0.1 and $10 \mathrm{~cm}^{2} / \mathrm{g}$ at velocity $v=30 \mathrm{~km} / \mathrm{s}$ relevant for solving the core-cusp problem and the too-big-too-fail problem.

and the too-big-too-fail problems. On the other hand if the cross-section is too large the effects could be seen on larger scales and would be ruled out.

The limits on this cross-section come from comparing observations to simulations. One obvious constraint is from the Bullet cluster which gives an upper limit on the crosssection, $\sigma_{T} / M_{\mathrm{DM}}<1.25 \mathrm{~cm}^{2} / \mathrm{g}$ [77]. Since the transfer cross-section is very strongly velocity-dependent, it is important that this bound is imposed in the relevant velocity range $v \sim 1000 \mathrm{~km} / \mathrm{s}$. There are also constraints of $\sigma_{T} / M_{\mathrm{DM}} \lesssim 0.1$ to $1 \mathrm{~cm}^{2} / \mathrm{g}$ from Milky Way scales in the velocity range of $200 \mathrm{~km} / \mathrm{s}$ [70].

To solve the too-big-to-fail problem one needs a cross-section of the order of $\sigma_{T} / M_{\mathrm{DM}} \sim$ $0.1-10 \mathrm{~cm}^{2} / \mathrm{g}$ [69-71] at the velocity scale of drwarf galaxies $(v \sim 10-30 \mathrm{~km} / \mathrm{s})$. Comparing this to the limits from larger scale structures one finds that there might be a small region of parameter space left for a theory with velocity independent cross-section of around $\sigma_{T} / M_{\mathrm{DM}} \sim 0.6 \mathrm{~cm}^{2} / \mathrm{g}[69,70]$

In this paper we consider a velocity dependent cross-section where if the cross-section is around $1 \mathrm{~cm}^{2} / \mathrm{g}$ at velocities of $v \sim 10-30 \mathrm{~km} / \mathrm{s}$ it will be much smallerat the velocities relevant for the shapes of galaxies or the bullet cluster. Therefore there is no contradiction between the cross-sections needed to solve the too-big-too-fail problem and the constraints from the elipticity of galaxies.

In figure 7 we show the region in the parameter space of our model, where the transfer cross-section for Vector Dark Matter is in the desired region $\sigma_{T} / M_{\mathrm{DM}}=0.1-10 \mathrm{~cm}^{2} / \mathrm{g}$ at $v-$ $30 \mathrm{~km} / \mathrm{s}$ which can help solving these problems with dwarf galaxies. This is superimposed with the contours of the relic density for Vector Dark Matter in our model. In figure 8 we overlay this with the contours of $\sigma_{T} / m_{\mathrm{DM}}=1$ at other velocities. It readily follows from 


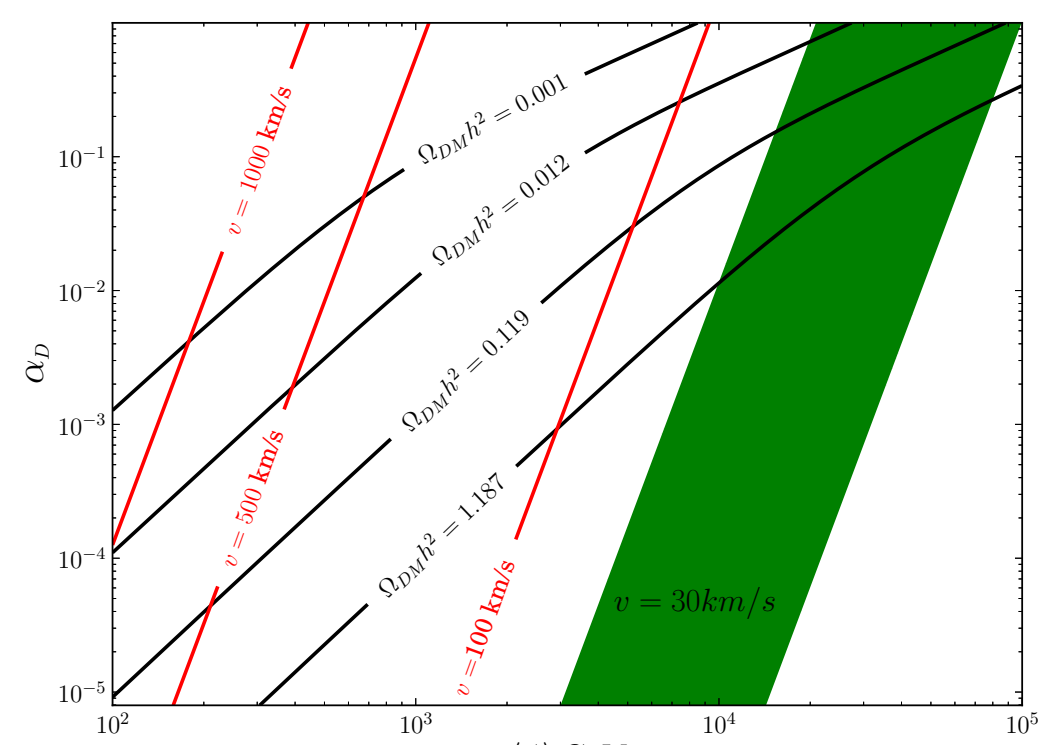

$\langle\phi\rangle \mathrm{GeV}$

Figure 8. Same as in figure 7 but with additional contours (in red) showing $\sigma_{T} / m_{\mathrm{DM}}=1$ at higher velocities: $v=100 \mathrm{~km} / \mathrm{s}, v=500 \mathrm{~km} / \mathrm{s}$ and $v=1000 \mathrm{~km} / \mathrm{s}$.

these considerations that the upper bound constraint from the Milky Way and from Bullet cluster at $v \sim 200$ to $\mathrm{km} / \mathrm{s}$ are satisfied by the self-interacting VDM in the regime where the relic density is in agreement with observations and the dwarf-galaxy-scale problems are addressed.

Monopoles dark matter self-interactions are obtained by replacing the electric with the magnetic Coulomb law, $\alpha_{D} \rightarrow \alpha_{\mathrm{mD}}=1 / \alpha_{D}$, which gives the limits seen in figures 9 and 10 for Monopole Dark Matter produced in a model with a second order phase transition.

\section{Conclusions}

We have shown that both dark monopoles and dark vector bosons can contribute to and accommodate the observed relic density and that the dark long range forces acting upon them correctly satisfy the observational constraints on $N_{\text {eff }}$ and on the transfer crosssection at large velocities. At the same time, the self-interacting Vector and Monopole DM intrinsic to our model produce the right size of transfer cross-sections relevant for addressing problems with dwarf galaxies. It would be interesting to use these general features and ingredients in simulations for formation and evolution of structure from the dwarf galaxies scale to the large scale.

\section{Acknowledgments}

We would like to thank Chris McCabe for useful discussions and comments. This material is based upon work supported by STFC through the IPPP grant ST/G000905/1. VVK 


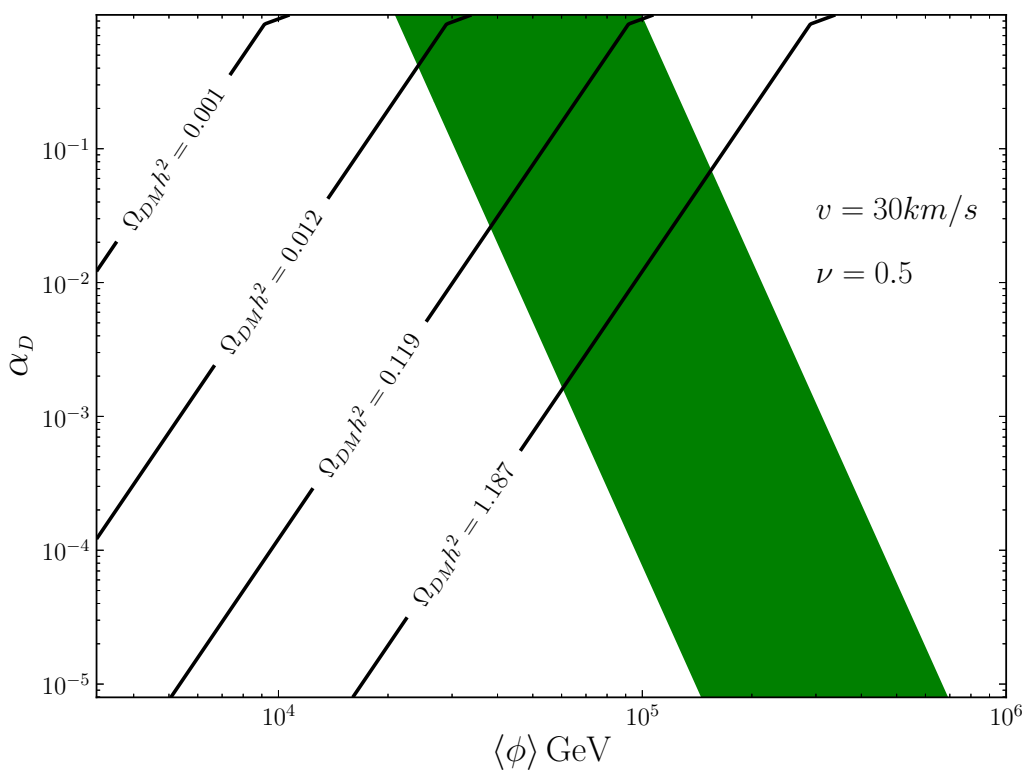

Figure 9. Monopole Dark Matter transfer cross-section and the relic density contours for the critical exponent $\nu=0.5$. The region in green shows the region in parameter space where $\sigma_{T} / m_{\mathrm{DM}}$ is in the interval between 0.1 and $10 \mathrm{~cm}^{2} / \mathrm{g}$ at velocity $v=30 \mathrm{~km} / \mathrm{s}$ relevant for solving the core-vs-cusp problem and the too-big-too-fail problem.

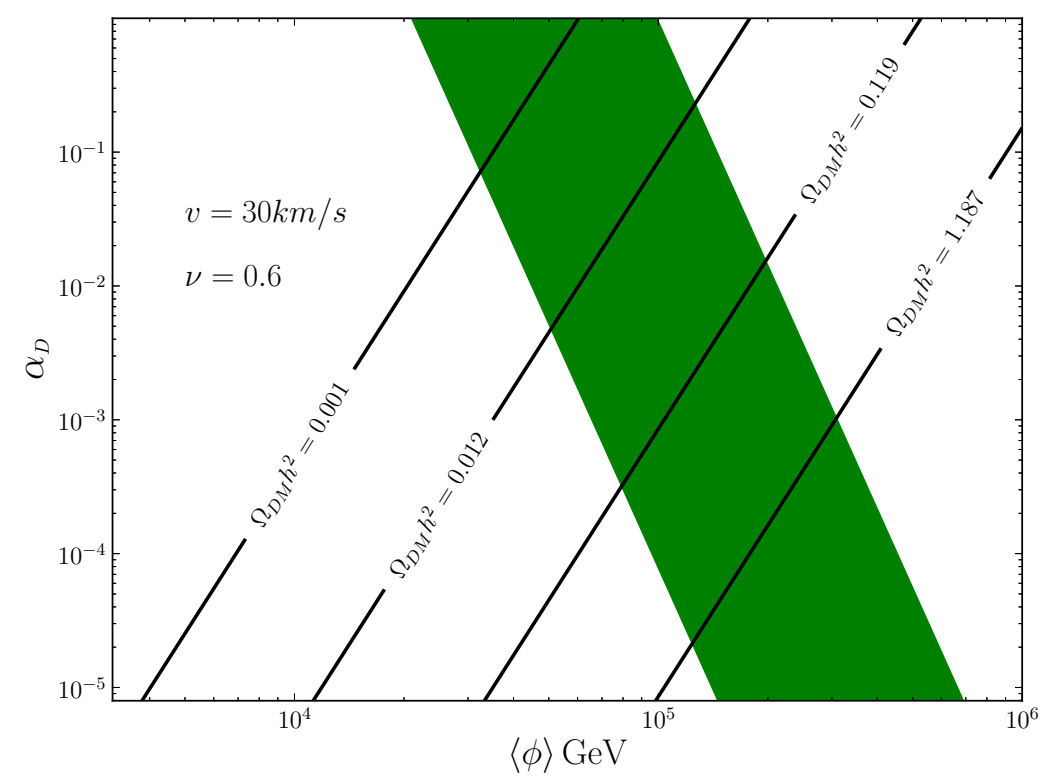

Figure 10. Monopole Dark Matter as in figure 9 with the critical exponent value $\nu=0.6$. 
acknowledges the support of the Wolfson Foundation and Royal Society through a Wolfson Research Merit Award. GR acknowledges the receipt of a Durham Doctoral Studentship.

\section{A Coleman-Weinberg with an adjoint scalar}

Consider a CSI SU $(2)_{D} \times \mathrm{SM}$ model with a scalar adjoint field in the hidden sector. The classically massless $\mathrm{SU}(2)$ theory with an adjoint scalar (1.1) was in fact one of the examples considered in the original paper of Coleman and Weinberg [13]. In a gauge where $\phi_{1,2}=$ $0, \phi_{3}=\phi$ they find a contribution from the gauge bosons to the effective potential of the form,

$$
V_{W^{\prime}}=\frac{3 g_{D}^{4}}{32 \pi^{2}} \phi^{4}\left(\log \frac{\phi^{2}}{\langle\phi\rangle^{2}}-\frac{25}{6}\right) .
$$

This is twice the result of the Abelian $\mathrm{U}(1)$ case as there now two massive vector bosons, $W_{ \pm}^{\prime}$. This is also to be compared with the case of $\mathrm{SU}(2)_{D}$ with a fundamental scalar considered in [8], where all three gauge bosons got a mass. Combining the 1-loop expression (A.1) with tree level potential we get,

$$
V=\frac{\lambda_{\phi}}{4} \phi^{4}+\frac{3 g_{D}^{4}}{32 \pi^{2}} \phi^{4}\left(\log \frac{\phi^{2}}{\langle\phi\rangle^{2}}-\frac{25}{6}\right)
$$

which has a non-trivial minimum with a vev for $\phi$ when

$$
\lambda_{\phi}(\langle\phi\rangle)=\frac{11}{8 \pi^{2}} g_{D}^{4}(\langle\phi\rangle) .
$$

With the adjoint scalar having acquired a vev, the $\mathrm{SU}(2)_{D}$ gauge group is broken to $\left.\mathrm{U}(1)\right)_{D}$ and we end up with two massive gauge bosons $W_{ \pm}^{\prime}$ a massless gauge boson $\gamma^{\prime}$, and one massive scalar field $\phi=\phi_{3}$ neutral under $\left.\mathrm{U}(1)\right)_{D}$. The masses are given by:

$$
M_{W^{\prime}}=g_{D}\langle\phi\rangle, \quad m_{\phi}^{2}=\frac{3 g_{D}^{2}\langle\phi\rangle^{2}}{4 \pi^{2}} .
$$

We now include the effects of the portal coupling between $\phi$ and the Higgs. The scalar potential is now,

$$
V=\lambda_{H}|H|^{4}+\lambda_{\phi} \operatorname{Tr}\left(\Phi \Phi^{\dagger}\right)^{2}-\lambda_{\mathrm{P}}|H|^{2} \operatorname{Tr}\left(\Phi \Phi^{\dagger}\right) .
$$

When $\phi$ develops a vev the portal term acts as a negative mass term for the Higgs and triggers electroweak symmetry breaking. Some of the relations above also get contributions from $\lambda_{\mathrm{P}}$

$$
\begin{aligned}
\lambda_{\phi} & =\frac{11}{16 \pi^{2}} g_{\mathrm{CW}}^{4}+\lambda_{\mathrm{P}} \frac{v^{2}}{2\langle\phi\rangle^{2}} \quad \text { at } \quad \mu=\langle\phi\rangle, \\
m_{\phi}^{2} & =\frac{3 g_{\mathrm{CW}}^{2}\langle\phi\rangle^{2}}{4 \pi^{2}}+\lambda_{\mathrm{P}} v^{2} .
\end{aligned}
$$

The Higgs portal interaction is also responsible for the mixing between the SM Higgs $h$ and the $\phi$ scalar of the Dark sector,

$$
M^{2}=\left(\begin{array}{cc}
2 \lambda_{H} v^{2} & -\sqrt{2 \lambda_{\mathrm{P}} \lambda H} v^{2} \\
-\sqrt{2 \lambda_{\mathrm{P}} \lambda H} v^{2} & m_{\phi}^{2}
\end{array}\right) .
$$


Open Access. This article is distributed under the terms of the Creative Commons Attribution License (CC-BY 4.0), which permits any use, distribution and reproduction in any medium, provided the original author(s) and source are credited.

\section{References}

[1] G. 't Hooft, Magnetic Monopoles in Unified Gauge Theories, Nucl. Phys. B 79 (1974) 276 [INSPIRE].

[2] A.M. Polyakov, Particle Spectrum in the Quantum Field Theory, in 30 Years of the Landau Institute - Selected Papers, World Scientific Publishing Co. (1996), pg. 540 [ISBN: 978-981-02-2253-6, 978-981-4500-46-3] [JETP Lett. 20 (1974) 194] [Pisma Zh. Eksp. Teor. Fiz. 20 (1974) 430] [INSPIRE].

[3] M.J. Strassler and K.M. Zurek, Echoes of a hidden valley at hadron colliders, Phys. Lett. B 651 (2007) 374 [hep-ph/0604261] [INSPIRE].

[4] M. Pospelov, A. Ritz and M.B. Voloshin, Secluded WIMP Dark Matter, Phys. Lett. B 662 (2008) 53 [arXiv: 0711.4866] [INSPIRE].

[5] N. Arkani-Hamed, D.P. Finkbeiner, T.R. Slatyer and N. Weiner, A Theory of Dark Matter, Phys. Rev. D 79 (2009) 015014 [arXiv: 0810.0713] [InSPIRE].

[6] H. Murayama and J. Shu, Topological Dark Matter, Phys. Lett. B 686 (2010) 162 [arXiv: 0905.1720] [INSPIRE].

[7] S. Baek, P. Ko and W.-I. Park, Hidden sector monopole, vector dark matter and dark radiation with Higgs portal, arXiv:1311.1035 [INSPIRE].

[8] V.V. Khoze, C. McCabe and G. Ro, Higgs vacuum stability from the dark matter portal, JHEP 08 (2014) 026 [arXiv: 1403.4953] [INSPIRE].

[9] T. Hambye and A. Strumia, Dynamical generation of the weak and Dark Matter scale, Phys. Rev. D 88 (2013) 055022 [arXiv:1306.2329] [INSPIRE].

[10] C.D. Carone and R. Ramos, Classical scale-invariance, the electroweak scale and vector dark matter, Phys. Rev. D 88 (2013) 055020 [arXiv:1307.8428] [INSPIRE].

[11] E.B. Bogomolny, Stability of Classical Solutions, Sov. J. Nucl. Phys. 24 (1976) 449 [Yad. Fiz. 24 (1976) 861] [INSPIRE].

[12] J. Preskill, Magnetic Monopoles, Ann. Rev. Nucl. Part. Sci. 34 (1984) 461 [InSPIRE].

[13] S.R. Coleman and E.J. Weinberg, Radiative Corrections as the Origin of Spontaneous Symmetry Breaking, Phys. Rev. D 7 (1973) 1888 [inSPIRE].

[14] T.W.B. Kibble, Topology of Cosmic Domains and Strings, J. Phys. A 9 (1976) 1387 [INSPIRE].

[15] W.H. Zurek, Cosmological Experiments in Superfluid Helium?, Nature 317 (1985) 505 [INSPIRE].

[16] G.W. Anderson and L.J. Hall, The Electroweak phase transition and baryogenesis, Phys. Rev. D 45 (1992) 2685 [INSPIRE].

[17] M. Dine, R.G. Leigh, P.Y. Huet, A.D. Linde and D.A. Linde, Towards the theory of the electroweak phase transition, Phys. Rev. D 46 (1992) 550 [hep-ph/9203203] [INSPIRE].

[18] M. Quirós, Finite temperature field theory and phase transitions, hep-ph/9901312 [INSPIRE].

[19] W.A. Bardeen, On naturalness in the standard model, FERMILAB-CONF-95-391 [INSPIRE]. 
[20] R. Hempfling, The Next-to-minimal Coleman-Weinberg model, Phys. Lett. B 379 (1996) 153 [hep-ph/9604278] [INSPIRE].

[21] K.A. Meissner and H. Nicolai, Conformal Symmetry and the Standard Model, Phys. Lett. B 648 (2007) 312 [hep-th/0612165] [INSPIRE].

[22] W.-F. Chang, J.N. Ng and J.M.S. Wu, Shadow Higgs from a scale-invariant hidden U(1)s model, Phys. Rev. D 75 (2007) 115016 [hep-ph/0701254] [INSPIRE].

[23] R. Foot, A.B. Kobakhidze, K.L. McDonald and R.R. Volkas, Neutrino mass in radiatively-broken scale-invariant models, Phys. Rev. D 76 (2007) 075014 [arXiv:0706.1829] [INSPIRE].

[24] R. Foot, A.B. Kobakhidze, K.L. McDonald and R.R. Volkas, A Solution to the hierarchy problem from an almost decoupled hidden sector within a classically scale invariant theory, Phys. Rev. D 77 (2008) 035006 [arXiv:0709.2750] [inSPIRE].

[25] T. Hambye and M.H.G. Tytgat, Electroweak symmetry breaking induced by dark matter, Phys. Lett. B 659 (2008) 651 [arXiv:0707.0633] [INSPIRE].

[26] M. Holthausen, M. Lindner and M.A. Schmidt, Radiative Symmetry Breaking of the Minimal Left-Right Symmetric Model, Phys. Rev. D 82 (2010) 055002 [arXiv:0911.0710] [INSPIRE].

[27] S. Iso, N. Okada and Y. Orikasa, Classically conformal B - L extended Standard Model, Phys. Lett. B 676 (2009) 81 [arXiv: 0902. 4050] [INSPIRE].

[28] C. Englert, J. Jaeckel, V.V. Khoze and M. Spannowsky, Emergence of the Electroweak Scale through the Higgs Portal, JHEP 04 (2013) 060 [arXiv: 1301.4224] [INSPIRE].

[29] M. Farina, D. Pappadopulo and A. Strumia, A modified naturalness principle and its experimental tests, JHEP 08 (2013) 022 [arXiv: 1303.7244] [INSPIRE].

[30] M. Heikinheimo, A. Racioppi, M. Raidal, C. Spethmann and K. Tuominen, Physical Naturalness and Dynamical Breaking of Classical Scale Invariance, Mod. Phys. Lett. A 29 (2014) 1450077 [arXiv: 1304.7006] [INSPIRE].

[31] V.V. Khoze and G. Ro, Leptogenesis and Neutrino Oscillations in the Classically Conformal Standard Model with the Higgs Portal, JHEP 10 (2013) 075 [arXiv:1307.3764] [INSPIRE].

[32] V.V. Khoze, Inflation and Dark Matter in the Higgs Portal of Classically Scale Invariant Standard Model, JHEP 11 (2013) 215 [arXiv: 1308.6338] [INSPIRE].

[33] E. Gabrielli, M. Heikinheimo, K. Kannike, A. Racioppi, M. Raidal and C. Spethmann, Towards Completing the Standard Model: Vacuum Stability, EWSB and Dark Matter, Phys. Rev. D 89 (2014) 015017 [arXiv: 1309.6632] [INSPIRE].

[34] M. Holthausen, J. Kubo, K.S. Lim and M. Lindner, Electroweak and Conformal Symmetry Breaking by a Strongly Coupled Hidden Sector, JHEP 12 (2013) 076 [arXiv:1310.4423] [INSPIRE].

[35] C.T. Hill, Is the Higgs Boson Associated with Coleman-Weinberg Dynamical Symmetry Breaking?, Phys. Rev. D 89 (2014) 073003 [arXiv: 1401.4185] [InSPIRE].

[36] J. Guo and Z. Kang, Higgs Naturalness and Dark Matter Stability by Scale Invariance, arXiv: 1401.5609 [INSPIRE].

[37] M. Hashimoto, S. Iso and Y. Orikasa, Radiative Symmetry Breaking from Flat Potential in various U(1)' models, Phys. Rev. D 89 (2014) 056010 [arXiv: 1401.5944] [INSPIRE].

[38] A. Salvio and A. Strumia, Agravity, JHEP 06 (2014) 080 [arXiv:1403.4226] [InSPIRE].

[39] K. Allison, C.T. Hill and G.G. Ross, Ultra-weak sector, Higgs boson mass and the dilaton, arXiv: 1404.6268 [INSPIRE]. 
[40] C. Tamarit, Higgs vacua behind barriers, arXiv:1404.7673 [INSPIRE].

[41] J. Kubo, K.S. Lim and M. Lindner, Gamma-ray Line from Nambu-Goldstone Dark Matter in a Scale Invariant Extension of the Standard Model, JHEP 09 (2014) 016 [arXiv:1405.1052] [INSPIRE].

[42] K. Kannike, A. Racioppi and M. Raidal, Embedding inflation into the Standard Model More evidence for classical scale invariance, JHEP 06 (2014) 154 [arXiv:1405.3987] [INSPIRE].

[43] M. Lindner, S. Schmidt and J. Smirnov, Neutrino Masses and Conformal Electro-Weak Symmetry Breaking, arXiv:1405.6204 [INSPIRE].

[44] C.G. Sanchez and B. Holdom, Monopoles, strings and dark matter, Phys. Rev. D 83 (2011) 123524 [arXiv: 1103.1632] [INSPIRE].

[45] W. Fischler and W. Tangarife Garcia, Hierarchies of SUSY Splittings and Invisible Photinos as Dark Matter, JHEP 01 (2011) 025 [arXiv:1011.0099] [inSPIRE].

[46] J. Evslin and S.B. Gudnason, Dwarf Galaxy Sized Monopoles as Dark Matter?, arXiv: 1202.0560 [INSPIRE].

[47] J.L. Feng, H. Tu and H.-B. Yu, Thermal Relics in Hidden Sectors, JCAP 10 (2008) 043 [arXiv:0808.2318] [INSPIRE].

[48] Planck collaboration, P.A.R. Ade et al., Planck 2013 results. XVI. Cosmological parameters, accepted in Astron. Astrophys. (2014) [arXiv:1303.5076] [INSPIRE].

[49] C. Brust, D.E. Kaplan and M.T. Walters, New Light Species and the CMB, JHEP 12 (2013) 058 [arXiv: 1303. 5379] [INSPIRE].

[50] A. Sommerfeld, Über die Beugung und Bremsung der Elektronen, Ann. Phys. 403 (1931) 257.

[51] J. Hisano, S. Matsumoto and M.M. Nojiri, Unitarity and higher order corrections in neutralino dark matter annihilation into two photons, Phys. Rev. D 67 (2003) 075014 [hep-ph/0212022] [INSPIRE].

[52] J. Hisano, S. Matsumoto and M.M. Nojiri, Explosive dark matter annihilation, Phys. Rev. Lett. 92 (2004) 031303 [hep-ph/0307216] [INSPIRE].

[53] J. Hisano, S. Matsumoto, M.M. Nojiri and O. Saito, Non-perturbative effect on dark matter annihilation and gamma ray signature from galactic center, Phys. Rev. D 71 (2005) 063528 [hep-ph/0412403] [INSPIRE].

[54] M. Cirelli, A. Strumia and M. Tamburini, Cosmology and Astrophysics of Minimal Dark Matter, Nucl. Phys. B 787 (2007) 152 [arXiv:0706.4071] [INSPIRE].

[55] L. Ackerman, M.R. Buckley, S.M. Carroll and M. Kamionkowski, Dark Matter and Dark Radiation, Phys. Rev. D 79 (2009) 023519 [arXiv:0810.5126] [INSPIRE].

[56] E.W. Kolb and M.S. Turner, The Early Universe, Frontiers in Physics (Book 69), Westview Press (1994) [ISBN: 0201626748, 9780201626742] [Nature 294 (1981) 521] [Phys. Today 44 (1991) 106] [INSPIRE].

[57] A.H. Guth and E.J. Weinberg, Could the Universe Have Recovered from a Slow First Order Phase Transition?, Nucl. Phys. B 212 (1983) 321 [INSPIRE].

[58] V.M. Ruutu, V.B. Eltsov, M. Krusius, Y. Makhlin, B. Placais and G.E. Volovik, Defect Formation in Quench-Cooled Superfluid Phase Transition, Phys. Rev. Lett. 80 (1998) 1465 [INSPIRE]. 
[59] M.J. Bowick, L. Chandar, E.A. Schiff and A.M. Srivastava, The Cosmological Kibble mechanism in the laboratory: String formation in liquid crystals, Science 263 (1994) 943 [hep-ph/9208233] [INSPIRE].

[60] Y.B. Zeldovich and M.Y. Khlopov, On the Concentration of Relic Magnetic Monopoles in the Universe, Phys. Lett. B 79 (1978) 239 [InSPIRE].

[61] J. Preskill, Cosmological Production of Superheavy Magnetic Monopoles, Phys. Rev. Lett. 43 (1979) 1365 [inSPIRE].

[62] A. Vilenkin and E.P.S. Shellard, Cosmic Strings and Other Topological Deffects, Cambridge University Press (1994).

[63] D.N. Spergel and P.J. Steinhardt, Observational evidence for selfinteracting cold dark matter, Phys. Rev. Lett. 84 (2000) 3760 [astro-ph/9909386] [INSPIRE].

[64] J.F. Navarro, C.S. Frenk and S.D.M. White, A Universal density profile from hierarchical clustering, Astrophys. J. 490 (1997) 493 [astro-ph/9611107] [INSPIRE].

[65] B. Moore, T.R. Quinn, F. Governato, J. Stadel and G. Lake, Cold collapse and the core catastrophe, Mon. Not. Roy. Astron. Soc. 310 (1999) 1147 [astro-ph/9903164] [InSPIRE].

[66] M.R. Buckley and P.J. Fox, Dark Matter Self-Interactions and Light Force Carriers, Phys. Rev. D 81 (2010) 083522 [arXiv:0911.3898] [INSPIRE].

[67] A. Loeb and N. Weiner, Cores in Dwarf Galaxies from Dark Matter with a Yukawa Potential, Phys. Rev. Lett. 106 (2011) 171302 [arXiv:1011.6374] [INSPIRE].

[68] M. Vogelsberger, J. Zavala and A. Loeb, Subhaloes in Self-Interacting Galactic Dark Matter Haloes, Mon. Not. Roy. Astron. Soc. 423 (2012) 3740 [arXiv:1201.5892] [INSPIRE].

[69] J. Zavala, M. Vogelsberger and M.G. Walker, Constraining Self-Interacting Dark Matter with the Milky Way's dwarf spheroidals, Mon. Not. Roy. Astron. Soc. 431 (2013) L20 [arXiv: 1211.6426] [INSPIRE].

[70] S. Tulin, H.-B. Yu and K.M. Zurek, Beyond Collisionless Dark Matter: Particle Physics Dynamics for Dark Matter Halo Structure, Phys. Rev. D 87 (2013) 115007 [arXiv:1302.3898] [INSPIRE].

[71] M.R. Buckley, J. Zavala, F.-Y. Cyr-Racine, K. Sigurdson and M. Vogelsberger, Scattering, Damping and Acoustic Oscillations: Simulating the Structure of Dark Matter Halos with Relativistic Force Carriers, Phys. Rev. D 90 (2014) 043524 [arXiv:1405.2075] [INSPIRE].

[72] J.L. Feng, M. Kaplinghat, H. Tu and H.-B. Yu, Hidden Charged Dark Matter, JCAP 07 (2009) 004 [arXiv: 0905.3039] [INSPIRE].

[73] D.E. Kaplan, G.Z. Krnjaic, K.R. Rehermann and C.M. Wells, Atomic Dark Matter, JCAP 05 (2010) 021 [arXiv: 0909.0753] [INSPIRE].

[74] D.E. Kaplan, G.Z. Krnjaic, K.R. Rehermann and C.M. Wells, Dark Atoms: Asymmetry and Direct Detection, JCAP 10 (2011) 011 [arXiv:1105.2073] [InSPIRE].

[75] S.A. Khrapak, A.V. Ivlev, G.E. Morfill and S.K. Zhdanov, Scattering in the attractive Yukawa potential in the limit of strong interaction, Phys. Rev. Lett. 90 (2003) 225002.

[76] S.A. Khrapak, A.V. Ivlev and G.E. Morfill, Momentum transfer in complex plasmas, Phys. Rev. E 70 (2004) 056405.

[77] S.W. Randall, M. Markevitch, D. Clowe, A.H. Gonzalez and M. Bradac, Constraints on the Self-Interaction Cross-Section of Dark Matter from Numerical Simulations of the Merging Galaxy Cluster 1E 0657-56, Astrophys. J. 679 (2008) 1173 [arXiv:0704.0261] [INSPIRE]. 